\title{
Solution of Extraordinary Differential Equations with Physical Reasoning by Obtaining Modal Reaction Series
}

\author{
Shantanu Das \\ Reactor Control Division, BARC, Mumbai 400085, India \\ Correspondence should be addressed to Shantanu Das, shantanu@barc.gov.in \\ Received 20 May 2009; Revised 14 December 2009; Accepted 22 February 2010 \\ Academic Editor: Andrzej Dzielinski \\ Copyright () 2010 Shantanu Das. This is an open access article distributed under the Creative Commons Attribution License, \\ which permits unrestricted use, distribution, and reproduction in any medium, provided the original work is properly cited. \\ Mathematical modeling of many engineering and physics problem leads to extraordinary differential equations like Nonlinear, \\ Delayed, and Fractional Order. An effective method is required to analyze the mathematical model which provides solutions \\ conforming to physical reality. A Fractional Differential Equation (FDE), where the leading differential operator is Riemann- \\ Liouvelli (RL) type requires fractional order initial states which are sometimes hard to physically relate. Therefore, we must \\ be able to solve these extraordinary systems, in space, time, frequency, area, volume, with physical reality conserved. Extra \\ Ordinary Differential equation Systems and its solution, with Physical Principle, of action-reaction and equivalent mathematical \\ decomposition method, are obtained as an aid for Physicists and Engineers to tackle the process dynamics with ease. This reactions- \\ chain generates internal modes from zeroth mode reaction to first mode second mode and to infinite modes; instantaneously in \\ parallel time or space-scales; and the sum of all these modes gives entire system reaction. This modal reaction as explained by \\ physics theory exactly matches the principle of Adomian Decomposition Method (ADM). Fractional Differential Equation (FDE) \\ with Riemann-Liouvelli formulation linear and non-linear is solved as per ADM. In this formulation of FDE by RL method it \\ is found that there is no need to worry about the fractional initial states; instead one can use integer order initial states (the \\ conventional ones) to arrive at solution of FDE. This new finding too is highlighted in this paper-along with several other problems \\ to give physical insight to the solution of extraordinary differential equation systems. This way one gets insight to Physics of General \\ Differential Equation Systems-and its solution-by Physical Principle and equivalent mathematical decomposition method. This \\ facilitates ease in modeling.
}

\section{Introduction}

General physics law states that a system will react to external stimulus and will have opposition to the changes; the process is described by system dynamic equations.

Let there be general differential equation system described as in

$$
\begin{aligned}
D_{x}^{m} u(x) & +a_{1} D_{x}^{m-1} u(x)+a_{2} D_{x}^{m-2} u(x)+\cdots \\
& +a_{m-1} D_{x}^{1} u(x)+a_{m} u(x)+b_{k}[u(x)]^{k} \\
& +b_{k-1}[u(x)]^{k-1}+\cdots b_{0} u(x)=G(x) .
\end{aligned}
$$

We can decompose this as Linear part

$$
L u(x)=G(x)-R(u)-N(u) .
$$

The operator $L$ represents a linear operator representing the highest orders of change in the process parameter. $L(u)=$ $d^{m} u(x) / d x^{m}=D_{x}^{m} u(x)$. This is easily invertible. This order of change $(m)$ could be one, two, or any positive integer or even fractional (say half, one fourth). This order of change could be with respect to time, space, space square (area) frequency, or time square depending on the process description. Where

$$
R(u)=a_{1} D_{x}^{m-1} u(x)+a_{2} D_{x}^{m-2} u(x)+\cdots+a_{m-1} D_{x}^{1} u(x)
$$

is the remainder differential operator of order less than $m$. This $R$ could be of integer or fractional order. The rest of the terms are put as (4) containing nonlinear as well as the linear 
terms, assuming (4) as analytic function $N$

$$
N(u)=a_{m} u(x)+b_{k}[u(x)]^{k}+b_{k-1}[u(x)]^{k-1}+\cdots b_{0} u(x) .
$$

The $G(x)$ is sum of all external stimuli source/sink.

General physics law states that a system will react to external stimulus and will have opposition to the changes, by the system reaction terms defined by $R$ and $N$ defined in the system description equation (1). These $R$ and $N$ generate internal stimulus when excited by external source/sink as to oppose the cause. The reactions are causal in nature. If the external stimulus and the internal reactions to the stimulus get balanced then the process parameter remains static without any growth (accumulation) or decay (loss). Else, the process parameter will have a solution as infinite (or finite) decomposed modes, generated by the system itself to oppose stimulus generated internally by the previous modes.

Adomian Decomposition Method (ADM), Computational method, yields analytical solution; it has certain advantages over standard numerical techniques [1-7]. However, the ADM was discovered in mid-late eighties and utilized to tackle non-linear problems of physics. ADM is free from rounding off errors as it does not involve discretization and does not require large computer memory. ADM is qualitative rather than quantitative, analytic, requiring neither linearization nor perturbation and continues with no resort to discretization. ADM splitting gives equation into linear $(L)$ that is containing the highest order of change, the remainder part $(R)$ that has change rates less than $(L)$, and then the non-linear $(N)$ part. Thereafter, inverting the "highest order" derivative $(L)$ in the linear operator on both sides of the differential equation is the first step. The second step is to identify the initial/boundary conditions and terms involving the independent variables alone, as initial approximation. Decomposing the unknown functions $(N)$ into series whose components are to be determined as ADM. The decomposed parts of ADM method are related physically to system reactions of various modes from zeroth mode to infinity mode. The sum of all these modes is the solution of Differential Equation (Nonlinear Linear Integer Order or Fractional Order). Physically the zeroth mode reaction comes from external stimulus plus the initial integer-order states; which instantly generates the internal stimuli of infinite modes, to oppose this first action (change), in an opposite way, in time or space (at the origin). The exact ADM mathematics generates these infinite modes reactions; therefore ADM is close to physical reality. The ADM helps to physically visualize the reaction of system by decomposing the total gross reaction into all these infinite modes. If the differential equation system with $(L)$ is of RiemannLiouvelli type fractional operator, then classically one needs the initial states as fractional order like $u^{\alpha}(0), u^{\alpha-1}(0)$, and so forth. These states are hard to visualize physically. With this $\mathrm{ADM}$, the RL formulation does not need these states instead $u(0), \dot{u}(0)$, the integer-order states, give the solution, being thus physically easily realizable. This new finding too is highlighted in this paper, along with several other problems to give physical insight to the solution of extraordinary differential equation systems. By this way, one gets insight to Physics of General Differential Equation Systems, and its solution, by Physical Principle and equivalent mathematical decomposition method. This provides ease in modeling systems close to physical reality. The system transfer function for analysis is delta function excitation (forcing function). This gives solution to homogeneous set of system of differential equations, called Green's function. Response to any other type of forcing function (say Heaviside's Step, Ramp or Sinusoidal function) is obtained by convoluting this delta function's response with (other) excitation function. Therefore most of the examples are discussed with delta function as forcing function; however the solution to any other type of forcing function is similar. One example is thus solved for mass-spring damper with (half) fractional-order element with Heaviside's step as input to demonstrate the utility of these methods.

\section{Physical Reasoning to Solve First-Order System and Its Mode Decomposition}

The application of ADM to simple ordinary differential equation will give insight to the action reaction theory of physics. Thus the ADM will try to explain the physical behavior too. It will be therefore demonstrated that ADM is actually translating the physics of the process, where any change is opposed by the system itself.

Consider the first order differential equation

$$
\begin{array}{r}
\dot{x}(t)+a x(t)=f(t) \text { or in term of }(1), \\
\text { as } D_{t}^{1} x(t)+a x(t)=f(t)
\end{array}
$$

with initial condition and forcing function (source) as $x(0)=$ $0, f(t)=K \delta(t)$. Practically, let $x(t)$ be instantaneous current of RC circuit connected to battery by a switch. The voltage excitation is a step function $V_{B B} h(t)$, where $h(t)=0 ; t<0$ and $h(t)=1 ; t \geq 0$ a Heaviside's step function. The circuit equation is

$$
\begin{gathered}
\frac{1}{C} \int_{0^{+}}^{t} i(t) d t+R i(t)=V_{B B} h(t) \quad \text { or } \\
\frac{1}{R C} \int_{0^{+}}^{t} i(t) d t+i(t)=\frac{V_{B B} h(t)}{R} .
\end{gathered}
$$

Differentiating (6), we get

$$
\frac{d}{d t} i(t)+\frac{1}{R C} i(t)=\frac{V_{B B}}{R} \delta(t) .
$$

Equation (6) is a voltage equation and (7) is current equation, rewritten with compliance with (5). This basic equation like (5) and (7) gives rate of change of current (function) as related to external stimulus. The current excitation is impulse excitation in (7). Have the initial current in the system be zero $i(0)=0$. This system has characteristic 
time constant $R C$ seconds; meaning that current in system changes $e$ times in $R C$ seconds. We shall consider response at larger time scales than $R C$. In (5) this characteristic time constant is $(1 / a)$. Here time scales and concept of time constant is mentioned. This could be length scale, frequencyscale time square, scale area volume, or any other scales depending on the units of $a$ in (5).

Initial time zero, at the switch closer instance, gives impulse excitation of current, assuming that if the capacitance of the circuit was absent, at this initial instant (capacitance comes into action at retarded time), then the current in the resistance is $i(t)=\left(V_{B B} / R\right) \int_{0}^{t} \delta(t) d t=$ $\left(V_{B B} / R\right) D_{t}^{-1} \delta(t)=\left(V_{B B} / R\right)$ a constant.

Here a point is mentioned that integration of the forcing function comes because the inertial element capacitance is present in the circuit and in (7). If the capacitor is completely absent then the current reaction will be simple $\left(V_{B B} / R\right) \delta(t)$, meaning that the current would have vanished instantaneously with the impulse and the current would be then zero, that is, $i(t)=0$ at $t \geq 0$. This initial moment current in resistor is $V_{B B} / R$ at $t=0$; as initially uncharged capacitor acts as short circuit impedance. The capacitor presence is making the current linger for time greater than zero. The circuit as natural reaction to any force will oppose this flow of current that is the change in current from zero to $V_{B B} / R$ is the capacitor action.

Therefore, the first (and the foremost) reaction comes from the resistive element (without lag or lead). That is $i_{0}=i(0)+D_{t}^{-1}\left[V_{B B} \delta(t) / R\right]=V_{B B} / R$, in the absence of the capacitive element (initially short circuited); this is the first reaction due to external force (and initial current if all will be present in the circuit). This sudden change in charges (Coulombs) cannot flow into capacitor, as the voltage across it cannot change instantaneously. This constant action of current gives rise to a rate of change of current in the system (per unit time constant) and is $\left(i_{0} / R C\right) \mathrm{A} / \mathrm{s}$. The first reactionary constant current thus is opposed by internal generated current as $i_{1}=-D_{t}^{-1}\left[i_{0} / R C\right]$, which is in opposition to this first reactionary current initial reaction, therefore negative. This action reaction summed up to give $i(t)=i_{0}+i_{i}$, the total current. The internally generated reactionary current gives a rate of change as $\left(i_{1} / R C\right) \mathrm{A} / \mathrm{s}$, which will generate opposition current to the cause $i_{1}$, as $i_{2}=-D_{t}^{-1}\left[i_{1} / R C\right]$, which again is added to give total reaction as $i(t)=i_{0}+i_{1}+i_{2}$, as the total current. By this way, infinite set of stimulus currents are generated as chain reaction giving the total current as $i(t)=\sum_{n=0}^{\infty} i_{0}$, where $i_{0}$ is the reaction due to external stimulus (and initial current if present in the circuit) and the rest are internally generated modes, acting in opposition to the rate of change in current.

The reaction $i(t)$, for $(7)$, can therefore be written as

$$
\begin{aligned}
i(t)= & {\left[i(0)+D_{t}^{-1}\left(\frac{V_{B B} \delta(t)}{R}\right)\right] } \\
& +\frac{1}{R C}(-1)^{n} D_{t}^{-1}\left[\sum_{n=1}^{\infty} i_{n-1}\right] .
\end{aligned}
$$

Equation (8) is appearing as physical reasoning and as infinite series as

$$
\begin{aligned}
i(t)= & i_{0}-\frac{1}{R C} D_{t}^{-1}\left[i_{0}\right]+\frac{1}{R C} D_{t}^{-1}\left[i_{1}\right] \\
& -\frac{1}{R C} D_{t}^{-1}\left[i_{2}\right]+\frac{1}{R C} D_{t}^{-1}\left[i_{3}\right]+\cdots .
\end{aligned}
$$

In recursion, we obtain

$$
\begin{aligned}
i_{0} & =i(0)+D_{t}^{-1}\left[\frac{V_{B B} \delta(t)}{R}\right], \\
i_{n} & =-\frac{1}{R C} D_{t}^{-1}\left[i_{n-1}\right] \quad n \geq 1 .
\end{aligned}
$$

Applying (10), we obtain

$$
\begin{gathered}
i_{0}=\frac{V_{B B}}{R}, \\
i_{1}=-\frac{1}{R C} D_{t}^{-1}\left[\frac{V_{B B}}{R}\right]=-\frac{V_{B B}}{R} \frac{t}{R C}, \\
i_{2}=-\frac{1}{R C} D_{t}^{-1}\left[-\frac{V_{B B}}{R} \frac{t}{R C}\right]=\frac{V_{B B}}{R} \frac{1}{2 !}\left(\frac{t}{R C}\right)^{2}, \\
i_{3}=-\frac{1}{R C} D_{t}^{-1}\left[\frac{V_{B B}}{R} \frac{1}{2 !}\left(\frac{t}{R C}\right)^{3}\right]=-\frac{V_{B B}}{R} \frac{1}{3 !}\left(\frac{t}{R C}\right)^{3} .
\end{gathered}
$$

Giving the total reaction of the system (7) as

$$
\begin{aligned}
i(t) & =\frac{V_{B B}}{R}\left(1-\frac{t}{R C}+\frac{1}{2 !}\left(\frac{t}{R C}\right)^{2}-\frac{1}{3 !}\left(\frac{t}{R C}\right)^{3}+\cdots\right) \\
& =\frac{V_{B B}}{R} e^{-(t / R C)} .
\end{aligned}
$$

The physical reasoning logic "opposite reaction to action" gives Mode Decomposition, and addition of all these infinite modes gives the entire system response. The observation is that zeroth mode reaction is formed by the external source/sink stimulus plus any initial condition. To oppose that rate of change, an opposite internal reaction integral action takes place. This internal action is the first-mode reaction which causes a rate of change; again integral action to this first mode, in opposition, makes the second-modal reaction and so on to make sum of "converging" analytical solution to the system's differential equation.

\section{Physical Reasoning to Solve Second-Order System and Its Mode Decomposition}

Consider a classical oscillator of integer-second order, mass spring system represented as

$$
m D_{t}^{2} x(t)+k x(t)=f(t)
$$

with initial conditions and forcing function defined as $x(0)=$ $0, \dot{x}(0)=0, f(t)=\delta(t)$. We can rewrite the equation as

$$
D_{t}^{2} x(t)=\ddot{x}(t)=\frac{1}{m} f(t)-\frac{k}{m} x(t) .
$$


The above equation gives insight to physical aspect of the process. The RHS states the opposing action to a forcing function, which is manifested as motion given by LHS of (14). At the initial time, the displacement being zero with the velocity implies that the displacement at just time $t=0^{+}$is due to the forcing function alone. This displacement action is without any opposition.

This (zeroth mode) displacement call is $x_{0}=$ $D_{t}^{-2}(1 / m) f(t)$. Due to the nature of this forcing function as an impulse, the displacement (zeroth mode) takes the form of $x_{0}=(1 / m) D_{t}^{-2} \delta(t)=(1 / m) t$. This displacement action would be true, in the absence of any retarding or opposing element say spring or friction. (In case of (14) it is spring action.) In absence of any opposition, the constitutive equation will be $m \ddot{x}(t)=f(t)$, and for impulse force, the displacement will be linear function of time $x(t)=t / m$, with constant velocity $\dot{x}(t)=(1 / m)$.

The presence of spring makes the equation of motion like: $\ddot{x}(t)=(1 / m) f(t)-(k / m) x(t)$; the external excitation is opposed by the spring action by opposite spring force and is internally generated $f_{i}=-(k / m) x_{i-1}$. The primary and the zeroth modes of displacement are due to external force on the mass that is $x_{0}=(1 / m) D_{t}^{-2} f(t)=(1 / m) D_{t}^{-2} \delta(t)=$ $(1 / m) t$. This zeroth mode of displacement is solely due to external excitation since the initial conditions are at rest. This displacement is now opposed by spring. Due to this opposing element, the displacement caused by external force, the spring generates an opposing force (first mode, from zeroth-order mode displacement), as $f_{1}=-(\mathrm{k} / \mathrm{m}) x_{0}$, and due to this new internal force, the displacement would be

$$
\begin{aligned}
x_{1} & =D_{t}^{-2} f_{1}=-\left(\frac{k}{m}\right) D_{t}^{-2} x_{0} \\
& =-\left(\frac{k}{m}\right) D_{t}^{-2}\left(\left(\frac{1}{m}\right) t\right)=-\left(\frac{k}{m^{2}}\right)\left(\frac{t^{3}}{3 !}\right),
\end{aligned}
$$

this is the first-order mode reaction displacement. This displacement again generates an internal force, inside the spring as $f_{2}=-(\mathrm{k} / \mathrm{m}) x_{1}=+\left(\mathrm{k}^{2} / \mathrm{m}^{3}\right)\left(t^{3} / 3 !\right)$, and to this force the displacement is second-order mode

$$
\begin{aligned}
x_{2} & =D_{t}^{-2} f_{2}=-\left(\frac{k}{m}\right) D_{t}^{-2} x_{1} \\
& =-\left(\frac{k}{m}\right) D_{t}^{-2}\left(-\left(\frac{k}{m}\right)\left(\frac{1}{m}\right)\left(\frac{t^{3}}{3 !}\right)\right) \\
& =\left(\frac{k^{2}}{m^{3}}\right)\left(\frac{t^{5}}{5 !}\right), \ldots
\end{aligned}
$$

In the absence of the spring, the opposing forces will be zero. We can call this as displacement as sum of all the modal displacements from zero to infinity modes, with zero modes being the only reaction to the bare excitation (if any initial displacement and velocity are present); all other modes are opposing reactions taking place in the spring. The modes can be tabulated as in Table 1, for unit mass and unit spring stiffness system of equation as described previously.

The process block diagram is represented in Figure 1, with $k=1$ and $m=1$.
Adding up all the modal displacements reactions, the solution to (13) is obtained as infinite series:

$$
\begin{aligned}
x(t) & =x_{0}+x_{1}+x_{2}+x_{3}+\cdots \\
& =\frac{1}{m} t-\frac{k}{m^{2}} \frac{t^{3}}{3 !}+\frac{k^{2}}{m^{3}} \frac{t^{5}}{5 !}-\frac{k^{3}}{m^{4}} \frac{t^{7}}{7 !} \\
& =\frac{1}{m}\left[t-\frac{k}{m} \frac{t^{3}}{3 !}+\frac{k^{2}}{m^{2}} \frac{t^{5}}{5 !}-\cdots\right] .
\end{aligned}
$$

Multiplying the above series by $\sqrt{\mathrm{k} / \mathrm{m}}$ and dividing by the same, we get

$$
\begin{aligned}
x(t) & =\frac{1}{\sqrt{k m}}\left[\sqrt{\frac{k}{m}} t-\left(\frac{k}{m}\right)^{3 / 2} \frac{t^{3}}{3 !}+\left(\frac{k}{m}\right)^{5 / 2} \frac{t^{5}}{5 !}-\cdots\right] \\
& =\frac{1}{\sqrt{k m}} \sin \left(\sqrt{\frac{k}{m}} t\right)
\end{aligned}
$$

This is oscillator with natural frequency $\omega=\sqrt{k / m}$ radians per seconds.

\section{Adomian Decomposition Fundamentals and Adomian Polynomials}

We symbolize the general differential equation as

$$
F u=G
$$

with $F$ being General non-linear Ordinary Differential Operator, this can also be Fractional Differential Operator also of Riemann-Liouvelli (RL) or Caputo type. This operator can be decomposed as

$$
F u=L u+R u+N u=G
$$

with $L$ being the Highest Order Derivative (Integer or Fractional Order) which is invertible, $R$ the Linear differential (remainder) operator of order less than that of $L$. This can also be fractional differential linear operator, $N$ the Nonlinear Part which will be decomposed into infinite sum of Adomian Polynomial. (This term can too be of linear or constant if decomposition is still valid.) For decomposition this needs to be analytic, $G$ the source term.

The decomposed equation can be rewritten as

$$
L u=G-R u-N u \text {. }
$$

Applying invert operator on both sides, we get

$$
u=\Phi+L^{-1} G-L^{-1}[R(u)]-L^{-1}[N(u)],
$$

where $\Phi$ is solution of the homogeneous equation $L u=$ 0 ; so that $L \Phi=0$. This comes from initial/boundary conditions. The LHS of (19) physically is the reaction of each component of physical system, with RHS of (19) representing source/sink or forcing term. For example, a mass spring and damper system have the constituent 
TABLE 1: Decomposing the action reaction of second-order mass-spring system.

\begin{tabular}{lcc}
\hline Mode & Force & Displacement \\
\hline 0 & $f_{0}=(1 / m) f(t)=(1 / m) \delta(t)$ & $x_{0}=D_{t}^{-2} f_{0}=(1 / m) t$ \\
Higher modes & Higher modal internal forces & Higher modal internal displacements \\
1 & $f_{1}=-(k / m) x_{0}=-\left(k / m^{2}\right) t$ & $x_{1}=D_{t}^{-2} f_{1}=-\left(k / m^{2}\right)\left(t^{3} / 3 !\right)$ \\
2 & $f_{2}=-(k / m) x_{1}=+\left(k^{2} / m^{3}\right)\left(t^{3} / 3 !\right)$ & $x_{2}=D_{t}^{-2} f_{2}=+\left(k^{2} / m^{3}\right)\left(t^{5} / 5 !\right)$ \\
3 & $f_{3}=-(k / m) x_{2}=-\left(k^{3} / m^{3}\right)\left(t^{5} / 5 !\right)$ & $x_{3}=D_{t}^{-2} f_{3}=-\left(k^{3} / m^{4}\right)\left(t^{7} / 7 !\right)$ \\
$\ldots$ & $\ldots$ & $\ldots$ \\
\hline
\end{tabular}

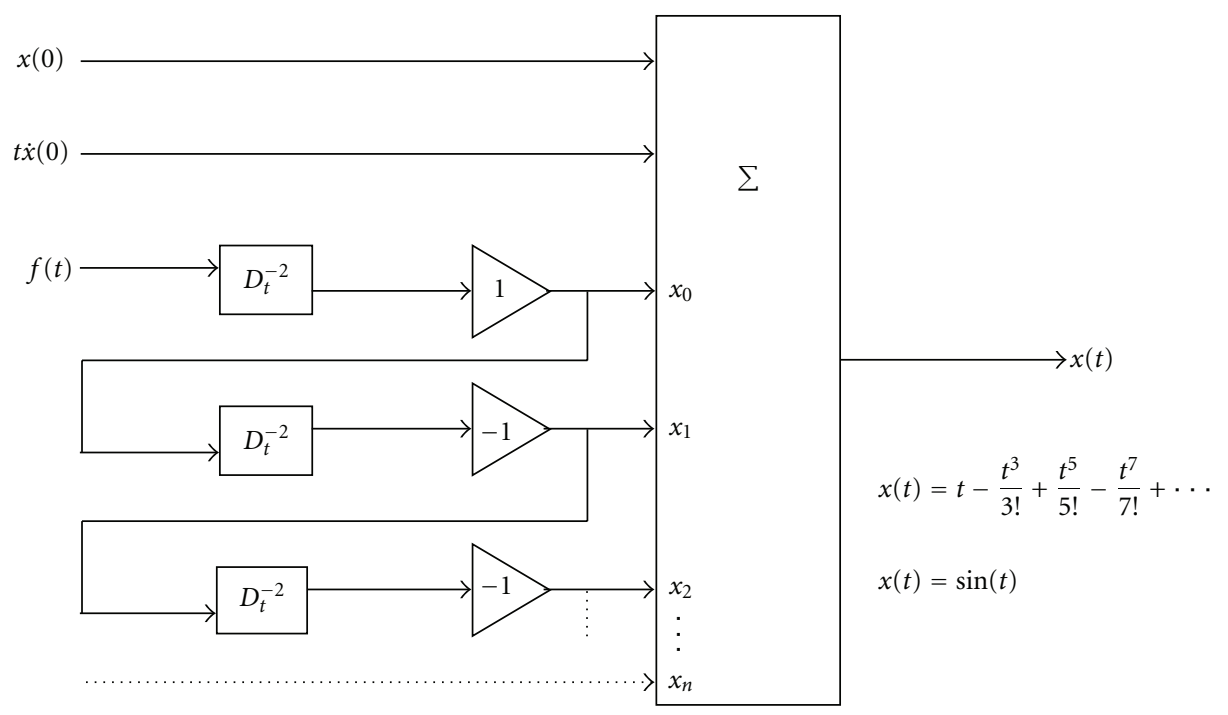

FIGURE 1: Block diagram showing decomposition and solution of second-order differential equation.

equation as $m\left(d^{2} x / d t^{2}\right)+c(d x / d t)+k x=f(t)$, the LHS of this is reaction of each elements, and the sum of which balances the RHS and the external force. In terms of (19) and (20), in this physical system $L=D_{t}^{2}, R=D_{t}^{1}$, and $N(x)=x$.

The solution to this is $x(t)=\Phi+(1 / m) L^{-1} f-$ $(\mathrm{c} / \mathrm{m}) L^{-1} R-(\mathrm{k} / \mathrm{m}) L^{-1} N$. In this example, the order of $L$ is two then $\Phi=u(0)+t \dot{u}(0)$. Assuming the time dependent differential equation system, the invert operator in this case is $L^{-1} f(t) \equiv D_{t}^{-2} f(t)=\iint_{0}^{t} f(t) d t d t$. If the order of $L$ is of order one then

$$
\Phi=u(0), \quad L^{-1} f(t) \equiv D_{t}^{-1} f(t)=\int_{0}^{t} f(t) d t
$$

For decomposition of the $N(u)$ part in (4) define a "grouping" parameter [2] close to one as $\lambda$. The function $u$ can be expressed as

$$
u(\lambda)=\sum_{n=0}^{\infty} \lambda^{n} u_{n}=u_{0}+\lambda u_{1}+\lambda^{2} u_{2}+\cdots
$$

Equation (24) is Maclurain series with respect to $\lambda$, with $u_{n}$ 's being coefficients of the Maclurain series around $\lambda=0$ that is $u_{n}=u^{(n)}(0) / n$ !. Then by $N(u)$ in Maclurain series with respect to $\lambda$, we obtain

$$
N(u)=\sum_{n=0}^{\infty} \lambda^{n} A_{n}
$$

where

$$
A_{n}=\frac{1}{n !}\left[\frac{d^{n}}{d \lambda^{n}} N\left(\sum_{k=0}^{\infty} \lambda^{k} u_{k}\right)\right]_{\lambda=0}
$$

The parameter $\lambda$ is just an identifier for collecting terms in a suitable way such that $u_{n}$ depends on $u_{0}, u_{1}, u_{2}, \ldots u_{n-1}$, and later on, we will set $\lambda=1$. Paremetrizing (22), we get

$$
u=\Phi+L^{-1} G-\lambda L^{-1}[R(u)]-\lambda L^{-1}[N(u)] .
$$

Expanding with decomposition (27), we obtain

$$
\begin{aligned}
u= & \sum_{n=0}^{\infty} \lambda^{n} u_{n}=\Phi+L^{-1} G-\lambda L^{-1} R\left(\sum_{n=0}^{\infty} \lambda^{n} u_{n}\right) \\
& -\lambda L^{-1} \sum_{n=0}^{\infty} \lambda^{n} A_{n} .
\end{aligned}
$$


Equating the coefficients of equal powers of $\lambda$ in the expression for $n=0$, to get $u_{0}$, then $n=1$, to get $u_{1}$ and so on, in (28), we get

$$
\begin{aligned}
& u_{0}=\Phi+L^{-1} G, \\
& u_{1}=-L^{-1}\left[R\left(u_{0}\right)\right]-L^{-1}\left(A_{0}\right), \\
& u_{2}=-L^{-1}\left[R\left(u_{1}\right)\right]-L^{-1}\left(A_{1}\right), \\
& \vdots \\
& u_{n}=-L^{-1}\left[R\left(u_{n-1}\right)\right]-L^{-1}\left(A_{n-1}\right), \quad n \geq 1 .
\end{aligned}
$$

Finally $\phi_{N}(t)=\sum_{n=0}^{N-1} u_{n}(t)$ with $N \geq 1$; exact solution of (19) is $u(t)=\lim _{N \rightarrow \infty} \phi_{N}(t)$. This method is applied in various problems of physics [8-15]. The convergence of this method is very well proved in [16-18].

In the described ADM method [1-7] expression (29) contains Adomian polynomials $A_{n}$ as recurring formulations, where the invert operator is operational. The finding of these $A_{n}$, from (26), is demonstrated here in this section. Suppose that the non-linear part of (20), that is, $N(u)$, is represented as $\sum_{n=0}^{\infty} \lambda^{n} A_{n}$; that is, $N(u(\lambda))$ is assumed to be analytic in $\lambda$. So we write $N u=N(u(\lambda))=$ $\sum_{n=0}^{\infty} \lambda^{n} A_{n}$. The $A_{n}$ 's are polynomial defined in such a way that each $A_{n}$ depends only on $u_{0}, u_{1}, u_{2}, \ldots u_{n}$. Thus, $A_{0}=$ $A_{0}\left(u_{0}\right), A_{1}=A_{1}\left(u_{0}, u_{1}\right), A_{2}=A_{2}\left(u_{0}, u_{1}, u_{2}\right)$, and so forth. Therefore, one possible formulation is listed below [2]:

$$
\begin{aligned}
A_{0}= & A_{0}\left(u_{0}\right)=N(u(\lambda))_{\lambda=0} \\
= & N\left(u_{0}+\lambda u_{1}+\lambda^{2} u_{2}+\cdots\right)_{\lambda=0}=N\left(u_{0}\right) \\
A_{1}= & A_{1}\left(u_{0}, u_{1}\right)=\left(\frac{\partial N}{\partial u}\right)\left(\frac{\partial u}{\partial \lambda}\right)_{\lambda=0}, \\
A_{2}= & A_{2}\left(u_{0}, u_{1}, u_{2}\right) \\
= & \frac{1}{2}\left[\left(\frac{\partial^{2} N}{\partial u^{2}}\right)\left(\frac{\partial u}{\partial \lambda}\right)^{2}+\left(\frac{\partial N}{\partial u}\right)\left(\frac{\partial^{2} u}{\partial \lambda^{2}}\right)\right]_{\lambda=0}, \\
A_{3}= & A_{3}\left(u_{0}, u_{1}, u_{2}, u_{3}\right) \\
= & \frac{1}{6}\left[\left(\frac{\partial^{3} N}{\partial u^{3}}\right)\left(\frac{\partial u}{\partial \lambda}\right)^{3}\right. \\
& \left.+3\left(\frac{\partial^{2} N}{\partial u^{2}}\right)\left(\frac{\partial u}{\partial \lambda}\right)\left(\frac{\partial^{2} u}{\partial \lambda^{2}}\right)+\left(\frac{\partial N}{\partial u}\right)\left(\frac{\partial^{3} u}{\partial \lambda^{3}}\right)\right]_{\lambda=0}
\end{aligned}
$$

The $A_{n}$ 's can be reformatted from (30) in the following form:

$$
\begin{aligned}
A_{0}= & N\left(u_{0}\right), \\
A_{1}= & u_{1}\left(\frac{d}{d u_{0}}\right) N\left(u_{0}\right)=u_{1} N^{\prime}\left(u_{0}\right), \\
A_{2}= & u_{2}\left(\frac{d}{d u_{0}}\right) N\left(u_{0}\right)+\frac{u_{1}^{2}}{2 !}\left(\frac{d^{2}}{d u_{0}^{2}}\right) N\left(u_{0}\right) \\
= & u_{2} N^{\prime}\left(u_{0}\right)+\frac{1}{2 !} u_{1}^{2} N^{\prime \prime}\left(u_{0}\right), \\
A_{3}= & u_{3}\left(\frac{d}{d u_{0}}\right) N\left(u_{0}\right)+u_{1} u_{2}\left(\frac{d^{2}}{d u_{0}^{2}}\right) N\left(u_{0}\right) \\
& +\frac{u_{1}^{3}}{3 !}\left(\frac{d^{3}}{d u_{0}^{3}}\right) N\left(u_{0}\right)=u_{3} N^{\prime}\left(u_{0}\right) \\
& +u_{1} u_{2} N^{\prime \prime}\left(u_{0}\right)+\frac{u_{1}^{3}}{3 !} N^{\prime \prime \prime}\left(u_{0}\right) .
\end{aligned}
$$

For the case where non-linear term is linear, that is to say $N(u)=u$; then $A_{n}=u_{n}$, else

$$
A_{n}=A_{n}\left(u_{0}, u_{1}, u_{2}, \ldots, u_{n}\right) \quad \forall n=0,1,2,3 \ldots
$$

For example, if $N(u)=u^{3}$, then Adomian Polynomials for this nonlinearity are

$$
\begin{gathered}
A_{0}=u_{0}^{3}, \\
A_{1}=3 u_{0}^{2} u_{1}, \\
A_{2}=3 u_{0}^{2} u_{2}+3 u_{1}^{2} u_{0}, \\
A_{3}=u_{1}^{3}+3 u_{0}^{2} u_{3}+6 u_{0} u_{1} u_{2}, \ldots
\end{gathered}
$$

The derivation of obtaining Adomian Polynomials comes from Generalized Taylor's series (Maclurain series) of several variables from linear analysis. This is described as follows:

$$
\begin{gathered}
N(u)=\sum_{n=0}^{\infty} \lambda^{n} A_{n}, \\
N\left(u_{0}+\lambda u_{1}+\lambda^{2} u_{2}+\cdots\right) \\
=A_{0}+\lambda A_{1}+\lambda^{2} A_{2}+\lambda^{3} A_{3}+\cdots .
\end{gathered}
$$

Put $\lambda=0$, to get $N\left(u_{0}\right)=A_{0}$.

Differentiate once (34), with respect to $\lambda$, to get

$$
\begin{aligned}
& \frac{d}{d \lambda}\left[N\left(u_{0}+\lambda u_{1}+\lambda^{2} u_{2}+\cdots\right)\right] \\
& =\frac{d}{d \lambda}\left(A_{0}+\lambda A_{1}+\lambda^{2} A_{2}+\cdots\right) .
\end{aligned}
$$

Using partial derivative expansion on the LHS of (35) and differentiating RHS of (35), we obtain the following:

$$
\begin{gathered}
\frac{\partial N\left(u_{0}+\lambda u_{1}+\lambda^{2} u_{2}+\cdots\right)}{\partial\left(u_{0}+\lambda u_{1}+\lambda^{2} u_{2}+\cdots\right)} \frac{\partial}{\partial \lambda}\left(u_{0}+\lambda u_{1}+\lambda^{2} u_{2}+\cdots\right) \\
=A_{1}+2 \lambda A_{2}+3 \lambda^{2} A_{3}+\cdots .
\end{gathered}
$$


Put $\lambda=0$ in the above (36) to get

$$
A_{1}=u_{1} \frac{d N\left(u_{0}\right)}{d u_{0}}=u_{1} N^{\prime}\left(u_{0}\right)
$$

Differentiating once more (37) with respect to $\lambda$, we get

$$
\begin{gathered}
\frac{d}{d \lambda}\left[\frac{\partial N\left(u_{0}+\lambda u_{1}+\cdots\right)}{\partial\left(u_{0}+\lambda u_{1}+\cdots\right)} \frac{\partial}{\partial \lambda}\left(u_{0}+\lambda u_{1}+\cdots\right)\right] \\
=\frac{d}{d \lambda}\left(A_{1}+2 \lambda A_{2}+3 \lambda^{2} A_{3}+\cdots\right) .
\end{gathered}
$$

Using partial derivative expansion on the LHS of (38) and differentiating RHS of (38), we obtain the following:

$$
\begin{aligned}
\frac{\partial}{\partial \lambda}\left(u_{0}+\lambda u_{1}+\cdots\right) \frac{d}{d \lambda}\left[\frac{\partial N\left(u_{0}+\lambda u_{1}+\cdots\right)}{\partial\left(u_{0}+\lambda u_{1}+\cdots\right)}\right] \\
+\frac{\partial N\left(u_{0}+\lambda u_{1}+\cdots\right)}{\partial\left(u_{0}+\lambda u_{1}+\cdots\right)} \frac{d}{d \lambda} \frac{\partial}{\partial \lambda} \\
\quad \times\left(u_{0}+\lambda u_{1}+\cdots\right)=2 A_{2}+3 ! \lambda A_{3}+\cdots .
\end{aligned}
$$

Using partial derivative expansion on the LHS of (39) and differentiating RHS of (39), we obtain the following:

$$
\begin{aligned}
\left(u_{1}+\right. & \left.2 \lambda u_{2}+\cdots\right) \frac{\partial^{2} N\left(u_{0}+\lambda u_{1}+\cdots\right)}{\partial\left(u_{0}+\lambda u_{1}+\cdots\right)^{2}} \frac{\partial}{\partial \lambda} \\
& \times\left(u_{0}+\lambda u_{1}+\cdots\right)+\frac{\partial N\left(u_{0}+\lambda u_{1}+\cdots\right)}{\partial\left(u_{0}+\lambda u_{1}+\cdots\right)} \\
& \times\left(2 u_{2}+3 \lambda u_{3}+\cdots\right)=2 A_{2}+\cdots
\end{aligned}
$$

Putting $\lambda=0$ in the above expression (40), we obtain

$$
u_{1}^{2} \frac{\partial^{2} N\left(u_{0}\right)}{\partial u_{0}^{2}}+2 u_{2} \frac{\partial N\left(u_{0}\right)}{\partial u_{0}}=2 A_{2}
$$

Implying that

$$
\begin{aligned}
A_{2} & =u_{2} \frac{d N\left(u_{0}\right)}{d u_{0}}+\frac{u_{1}^{2}}{2} \frac{d^{2} N\left(u_{0}\right)}{d u_{0}^{2}} \\
& =u_{2} N^{\prime}\left(u_{0}\right)+\frac{1}{2} u_{1}^{2} N^{\prime \prime}\left(u_{0}\right) .
\end{aligned}
$$

Continuing like this, we get set of the Adomian Polynomials for the function $N(u)$.

If the nonlinearity part is

$$
N_{1}(y)=y^{2}=\sum_{k=0}^{\infty} A_{n}
$$

then the Adomian Polynomials are

$$
\begin{gathered}
A_{0}=y_{0}^{2}, \\
A_{1}=2 y_{0} y_{1}, \\
A_{2}=y_{1}^{2}+2 y_{0} y_{2}, \\
A_{3}=2 y_{0} y_{3}+2 y_{0} y_{2} \ldots
\end{gathered}
$$

If the nonlinearity part is $N_{2}(y)=y^{3}=\sum_{k=0}^{\infty} B_{n}$ then the Adomian Polynomials are

$$
\begin{gathered}
B_{0}=y_{0}^{3}, \\
B_{1}=3 y_{0}^{2} y_{1}, \\
B_{2}=3 y_{0}^{2} y_{2}+3 y_{1}^{2} y_{0}, \\
B_{3}=3 y_{0}^{2} y_{3}+6 y_{0} y_{1} y_{2}+y_{1}^{3} \ldots
\end{gathered}
$$

For the linear term $N_{0}(y)=y=\sum_{k=0}^{\infty} y_{n}, A_{0}=y_{0}=x_{0}$ and $A_{n}=y_{n}$. For constant $N(u)$, the Adomian polynomials are $N_{0}(y)=K, A_{0}=N_{0}\left(y_{0}\right)=K, A_{1}=A_{2}=\cdots=A_{\infty}=0$. The series solution $\sum_{n=0}^{\infty} u_{n}$ thus may have finite terms with higher modes as zero, depending on $N(u)$.

\section{Generalization of Physical Law of Nature vis-à-vis ADM}

The physical description and then obtaining decomposed solution match well with the ADM. From Sections 2, 3, and 4, we generalize the system of General Differential Equations and give action-reaction laws to it so that one can obtain the solution by decomposition into infinite (or finite) modes.

Let there be general differential equation system as

$$
\begin{aligned}
D_{x}^{m} u(x) & +a_{1} D_{x}^{m-1} u(x)+a_{2} D_{x}^{m-2} u(x)+\cdots \\
& +a_{m} u(x)+b_{k}[u(x)]^{k}+b_{k-1}[u(x)]^{k-1} \\
& +\cdots b_{0} u(x)=G(x) .
\end{aligned}
$$

We can write (46) as

$$
L u(x)=G(x)-R(u)-N(u),
$$

where $R(u)=a_{1} D_{x}^{m-1}+a_{2} D_{x}^{m-2}+\cdots+a_{m-1} D_{x}^{1}$ is the remainder differential operator of order less than $m$. This $N(u)=a_{m} u(x)+b_{k}[u(x)]^{k}+b_{k-1}[u(x)]^{k-1}+\cdots b_{0} u(x)$ contains nonlinear as well as the linear terms. The $G(x)$ is sum of all external stimulus source/sink.

The $L$ represents a linear operator representing the highest orders of change in the process parameter. $L(u)=$ $d^{m} u(x) / d x^{m}=D_{x}^{m} u(x)$, which is easily invertible. This order of change $(m)$ could be one, two, or any positive integer or even fractional (say half, one fourth). This order of change could be with respect to time, space, space square (area) frequency, or time square, depending on the process 
description. Then with ADM we have

$$
\begin{aligned}
u(\lambda) & =u_{0}+\lambda u_{1}+\lambda^{2} u_{2}+\lambda^{3} u_{3}+\cdots, \\
u_{0} & =\Phi+L^{-1} G, \\
N[u(\lambda)] & =N\left(u_{0}+\lambda u_{1}+\lambda^{2} u_{2}+\cdots\right) \\
& =A_{0}+\lambda A_{1}+\lambda^{2} A_{2}+\lambda^{3} A_{3}+\cdots, \\
A_{0} & =N\left(u_{0}\right), \\
u_{1} & =-L^{-1} R\left(u_{0}\right)-L^{-1}\left(A_{1}\right), \\
A_{1} & =u_{1} N^{\prime}\left(u_{0}\right), \\
u_{2} & =-L^{-1} R\left(u_{1}\right)-L^{-1}\left(A_{1}\right), \\
A_{2} & =u_{2} N^{\prime}\left(u_{0}\right)+\frac{1}{2} u_{1}^{2} N^{\prime \prime}\left(u_{0}\right), \\
u_{3} & =-L^{-1} R\left(u_{2}\right)-L^{-1}\left(A_{2}\right), \\
A_{n-1} & =\frac{1}{(n-1) !}\left[\frac{d^{n-1} N\left(\sum_{k=0}^{\infty} \lambda^{k} u_{k}\right)}{d \lambda^{n-1}}\right]_{\lambda=0}, \\
u_{n} & =-L^{-1} R\left(u_{n-1}\right)-L^{-1}\left(A_{n-1}\right), \\
u(x) & =\sum_{n=0}^{\infty} u_{n} \cdot
\end{aligned}
$$

Equation (48) is exactly what was described in Sections 2 and 3 with physical principles of action reaction generating all internal modes.

\section{ADM Applied to First-Order Linear Differential Equation and Mode-Decomposition Solution}

Comparing with ADM described by (29) and (48) and with solution obtained by physical reasoning, to arrive at solution of (5), (7) gives a similarity. Therefore, the ADM is close to physical system behavior where the system reacts naturally in opposite way to resist any change-this is physical law, which is described by ADM, for solving system of differential equations (48).

The initial reaction to the external disturbances and the complete set of opposing reaction due to self-opposed elements to the change, get summed up to get the overall reaction yielding solution for (5) as set in (49) obtained visà-vis (48)

$$
\begin{gathered}
x(t)=x(0)+x_{0}+x_{1}+x_{2}+x_{3}+\cdots, \\
x(t)=x(0)+D_{t}^{-1} f(t)+a D_{t}^{-1} f_{1}(t)+a D_{t}^{-1} f_{2}(t)+\cdots, \\
x(t)=x(0)+D_{t}^{-1} f(t)+a \sum_{n=1}^{\infty}(-1)^{n}\left(D_{t}^{-1}\right)^{n} x_{n-1}, \\
x_{0}=x(0)+D_{t}^{-1} f(t), \\
x(t)=1-a t+\frac{(a t)^{2}}{2 !}-\frac{(a t)^{3}}{3 !}+\cdots=\exp (-a t) .
\end{gathered}
$$

The $x_{n}$ 's are internal reaction to internal generated forces for $n>0$. The action reaction process described is represented in Figure 2, with parameter $a=K=1$, the $D_{t}^{-1}$ is antiderivative operator of unity order is

$$
D_{t}^{-1} f(t) \equiv \int_{0}^{t} f(t) d t
$$

In the ADM, as described in (29) we can write the set of modes (reactions) as

$$
\begin{gathered}
x_{0}=\Phi+L^{-1} G, \\
x_{1}=-L^{-1}\left[R\left(x_{0}\right)\right]-L^{-1}\left(A_{0}\right), \\
x_{2}=-L^{-1}\left[R\left(x_{1}\right)\right]-L^{-1}\left(A_{1}\right) .
\end{gathered}
$$

In the case of $(5) L^{-1}=D_{t}^{-1}, G=f(t)=K \delta(t), N(x)=$ $a x$, is linear and $x_{0}=A_{0}$, with Adomian Polynomials (29) as: $A_{n}=a x_{n}$ with no remainder term as $R=0$. With this decomposition, we get

$$
\begin{gathered}
x_{0}=\Phi+L^{-1} G=D_{t}^{-1} K \delta(t)=K, \\
A_{0}=a x_{0}=a K \\
x_{1}=-L^{-1}\left[R\left(x_{0}\right)\right]-L^{-1}\left(A_{0}\right)=-D_{t}^{-1}[a K]=-K a t, \\
A_{1}=a x_{1}=-K a^{2} t, \\
x_{2}=-L^{-1}\left[R\left(x_{1}\right)\right]-L^{-1}\left(A_{1}\right)=-D_{t}^{-1}\left[-K a^{2} t\right]=K \frac{(a t)^{2}}{2 !}, \\
x_{3}=-L^{-1}\left[R\left(x_{2}\right)\right]-L^{-1}\left(A_{2}\right)=-D_{t}^{-1}\left[K a^{3} \frac{t^{2}}{2 !},\right. \\
x(t)=K\left[1-a t+\frac{t^{2}}{2 !}\right]=-K \frac{(a t)^{3}}{3 !}, \\
3 !
\end{gathered}
$$

The infinite currents at instant $(t \rightarrow 0)$ are formed. The first (or zeroth) reaction current is due to the initial state of the circuit and solely due to external forcerepresented by $x_{0}$. Then recurring opposite reactions occur as set of internal forces-due to opposing the changes, giving rise immediately the first mode, second mode (and to infinity modes) of currents; adding up giving the total current reaction as, $x(t)=x_{0}+\sum_{n=1}^{\infty} x_{n}$. Therefore, the ADM method is related to physical process of physics as to any "action" there is equal and opposite "reaction", may be external or internal to the system. In other words, all system reacts in opposite way to any change (external or internal).

\section{ADM Applied to Second-Order Linear Differential Equation System and Mode Decomposition}

The ADM method for (13) has $L^{-1}=D_{t}^{-2}, \Phi=x(0)+$ $t \dot{x}(0), G=(1 / m) f(t)=(1 / m) \delta(t), N(x)=(k / m) x$ which 


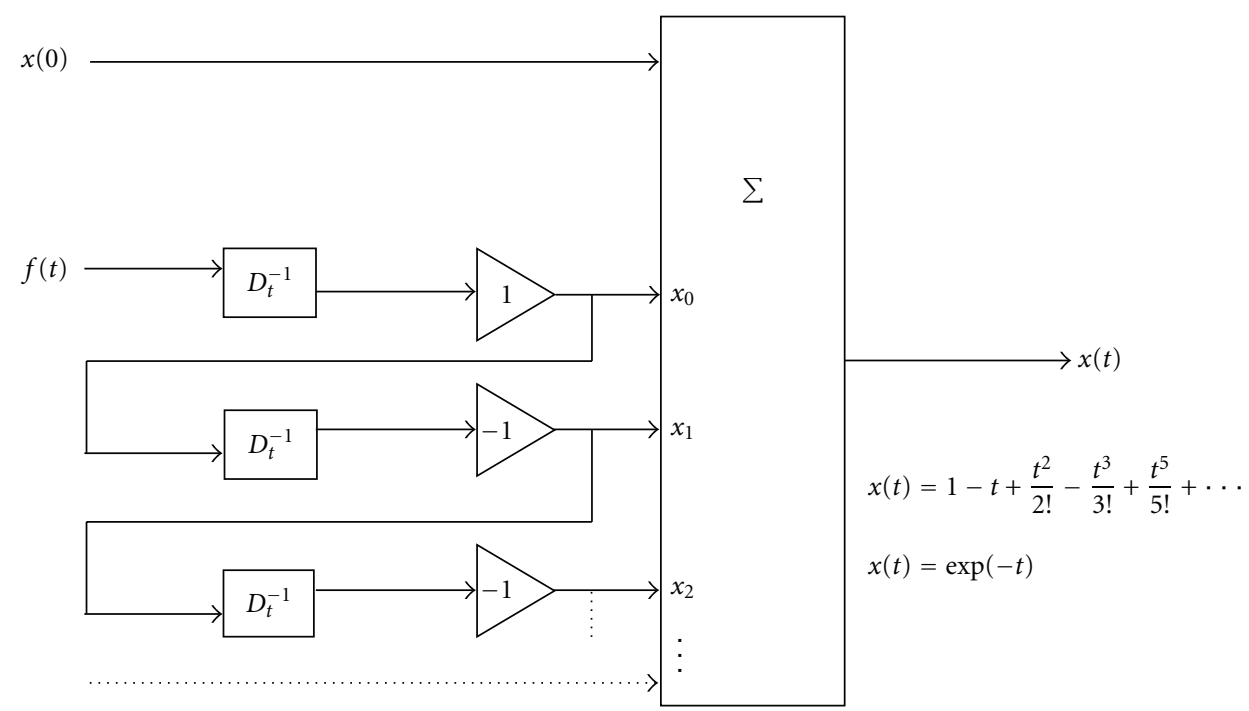

FIGURE 2: Block showing solution of first order differential equation by decomposition.

generates Adomian Polynomials (29) as $A_{0}=(k / m) x_{0}$, and $A_{n}=(\mathrm{k} / \mathrm{m}) x_{n}$. Here in (13) the remainder part is $R(x)=0$. Using the ADM for (13), one gets the modal displacements as described by physical reasoning also as

$$
\begin{gathered}
x_{0}=\Phi+L^{-1} G=D_{t}^{-2} \frac{1}{m} \delta(t)=\frac{1}{m} t, \\
A_{0}=\frac{k}{m} x_{0}=\frac{k}{m^{2}} t, \\
x_{1}=-L^{-1} R(x)-L^{-1}\left(A_{0}\right)=-D_{t}^{-2}\left[\frac{k}{m^{2}}\right]=-\frac{k}{m^{2}} \frac{t^{3}}{3 !}, \\
A_{1}=\frac{k}{m} x_{1}=-\frac{k^{2}}{m^{3}} \frac{t^{3}}{3 !}, \\
x_{2}=-L^{-1} R(x)-L^{-1}\left(A_{1}\right)=-D_{t}^{-2}\left(-\frac{t^{3}}{3 !}\right)=\frac{k^{2}}{m^{3}} \frac{t^{5}}{5 !}
\end{gathered}
$$

Giving the solution to (13) as

$$
\begin{aligned}
x(t) & =x_{0}+x_{1}+x_{2}+x_{3}+\cdots \\
& =\frac{1}{m} t-\frac{k}{m^{2}} \frac{t^{3}}{3 !}+\frac{k^{2}}{m^{3}} \frac{t^{5}}{5 !}-\frac{k^{3}}{m^{4}} \frac{t^{7}}{7 !}+\cdots+\cdots, \\
x(t) & =\frac{1}{m}\left[t-\frac{k}{m} \frac{t^{3}}{3 !}+\frac{k^{2}}{m^{2}} \frac{t^{5}}{5 !}-\cdots\right] .
\end{aligned}
$$

This too demonstrates the decomposition by ADM giving the physical modes of reaction process, generated as infinite series. Multiplying the above series by $\sqrt{\mathrm{k} / \mathrm{m}}$ and dividing by same we get

$$
\begin{aligned}
x(t) & =\frac{1}{\sqrt{k m}}\left[\sqrt{\frac{k}{m}} t-\left(\frac{k}{m}\right)^{3 / 2} \frac{t^{3}}{3 !}+\left(\frac{k}{m}\right)^{5 / 2} \frac{t^{5}}{5 !}-\cdots\right] \\
& =\frac{1}{\sqrt{k m}} \sin \left(\sqrt{\frac{k}{m}} t\right) .
\end{aligned}
$$

This is oscillator with natural frequency $\omega=\sqrt{k / m}$ radians per second; obtained earlier by physical law of actionreaction process.

\section{ADM for First-Order Linear Differential Equation System with Half-Order Element and Mode Decomposition}

Consider a first-order differential equation with the presence of a fractional (half) order element as

$$
\dot{x}(t)+D_{t}^{1 / 2} x(t)+x(t)=f(t)
$$

with initial conditions as $x(0)=0, f(t)=\delta(t)$.

The physical explanation as done in Sections 2 and 3 gives: the following solution:

$$
\begin{aligned}
x(t)= & x(0)+D_{t}^{-1} f(t) \\
& +\sum_{n=1}^{\infty}(-1)^{n}\left(D_{t}^{-1}+D_{t}^{-1 / 2}\right)^{n} f_{n-1}(t), \\
f_{0}= & x(0)+D_{t}^{-1} f(t) .
\end{aligned}
$$

For this system, the $R=D_{t}^{1 / 2}, L=D_{t}^{1}$ and nonlinear part is $N(x)=x$; (is linear in nature) thus as per (29) $A_{n}=x_{n}$. This demonstration also shows the fact that, if fractional order component is of lesser order than the leading order component (in this case is integer order of value one); then the initial condition is not dependent on the definition of the fractional derivative. Here the initial states are always of integer order in nature. 


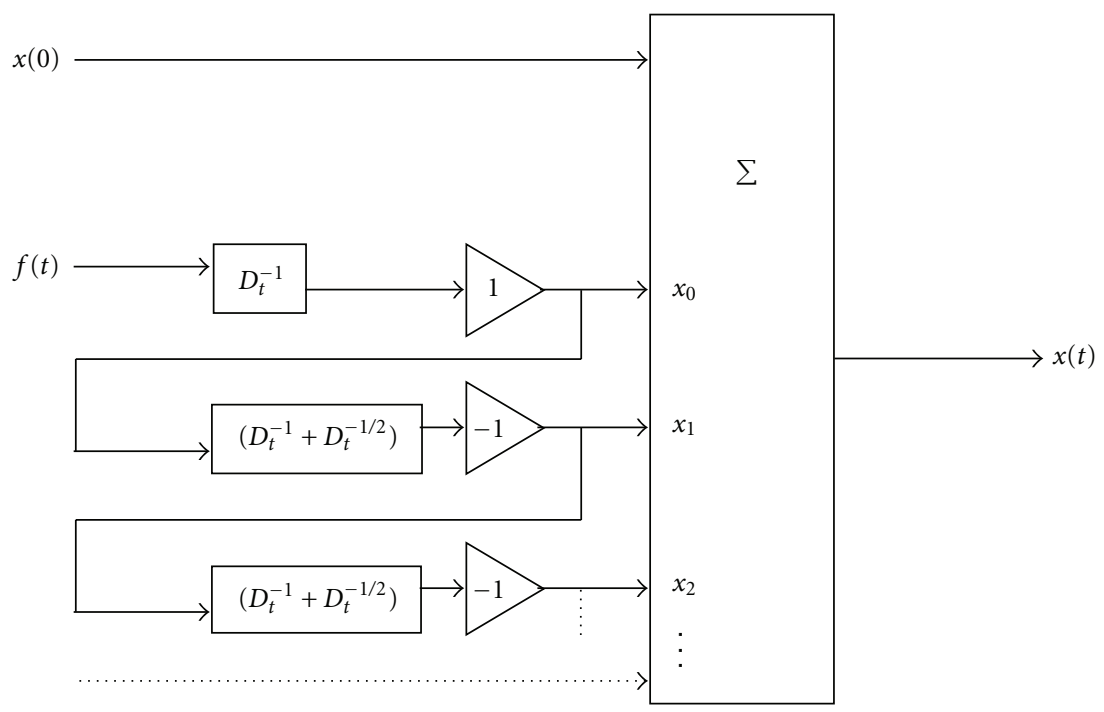

FIGURE 3: Block showing solution of first order differential equation by decomposition in presence of fractional half order term.

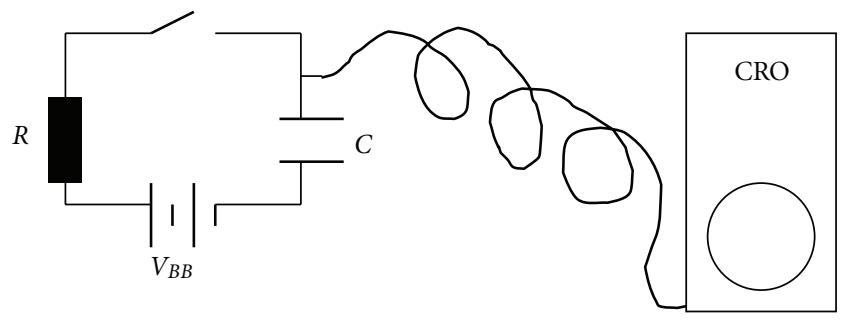

FIgURE 4: The RC circuit (a first order differential equation), with semi-infinite cable as fractional half order element.

The application of ADM method gives the components as, represented in Figure 3,

$$
\begin{aligned}
x_{0}= & \Phi+L^{-1} G=x(0)+D_{t}^{-1} f(t)=D_{t}^{-1} \delta(t)=1, \\
A_{0}= & x_{0}=1, \\
x_{1}= & -L^{-1}\left[R\left(x_{0}\right)\right]-L^{-1}\left[A_{0}\right]=-D_{t}^{-1}\left[D_{t}^{1 / 2}(1)\right] \\
& -D_{t}^{-1}[1]=-\frac{t^{1 / 2}}{\Gamma(1.5)}-t, \\
A_{1}= & x_{1}, \\
x_{2}= & -L\left[R\left(x_{1}\right)\right]-L^{-1}\left[A_{1}\right]=-D_{t}^{-1}\left[D^{1 / 2}\left(x_{1}\right)\right] \\
& -D_{t}^{-1}\left[x_{1}\right]=t+\frac{t^{3 / 2}}{\Gamma(2.5)}+\frac{2}{3} \frac{t^{3 / 2}}{\Gamma(1.5)}+\frac{t^{2}}{2} .
\end{aligned}
$$

The physics of this process may be viewed as, RC circuit reacting to an impulse current reaction in the presence of a semi-infinite RC cable (CRO Probe) connected to a shunt to measure the current. The semi-infinite cable acts as half order element [19] and the first-order circuit reaction thus will be modified by the presence of this half-order element. Refer Figure 4.

\section{ADM for Second-Order System with Half-Order Element and Its Physics}

9.1. Forcing Function as Delta Function. Solution of secondorder differential equation with presence of half order element $[19,20]$ is considered in

$$
\ddot{x}(t)+{ }_{0} D_{t}^{1 / 2} x(t)+x(t)=f(t) .
$$

With the initial condition as $x(0)=0, \dot{x}(0)=0, f(t)=\delta(t)$. Rearranging the above equation (59), we rewrite (double integrating both sides) as

$$
x(t)=x(0)+t \dot{x}(0)+D^{-2} f(t)-D^{-2} x(t)-D^{-3 / 2} x(t) .
$$

The physical reasoning generates

$$
\begin{gathered}
x_{0}=D_{t}^{-2} \delta(t)=t, \\
x_{1}=-\left(D_{t}^{-3 / 2}+D_{t}^{-2}\right) x_{0}=-\left(\frac{t^{5 / 2}}{\Gamma(7 / 2)}+\frac{t^{3}}{\Gamma(4)}\right), \\
x_{2}=-\left(D_{t}^{-3 / 2}+D_{t}^{-2}\right) x_{1}=\left(\frac{t^{4}}{\Gamma(5)}+2 \frac{t^{9 / 2}}{\Gamma(11 / 2)}+\frac{t^{5}}{\Gamma(6)}\right) .
\end{gathered}
$$

The modal displacements are generated after the application of external forcing function is depicted in the Table 2. The block diagram of the process is shown in Figure 5. 


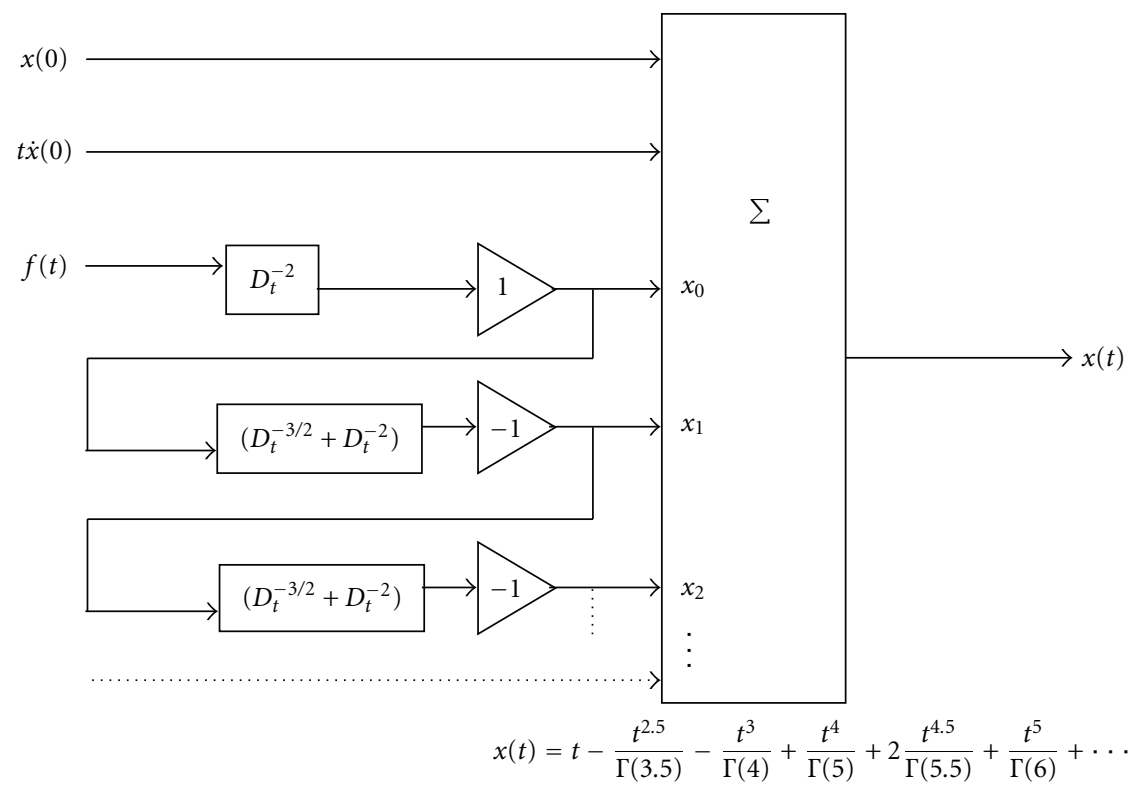

FIGURE 5: Block diagram showing solution of by decomposition of a second-order differential equation in presence of fractional-order term.

TABLE 2: Modal force and displacements for second-order system with fractional order damping.

\begin{tabular}{lcc}
\hline Mode & Force & Displacement \\
\hline 0 & $\delta(t)$ & $t$ \\
1 & $-t$ & $-\frac{t^{5 / 2}}{\Gamma(3.5)}-\frac{t^{3}}{\Gamma(4)}$ \\
2 & $\frac{t^{5 / 2}}{\Gamma(3.5)}+\frac{t^{3}}{\Gamma(4)}$ & $\frac{t^{4}}{\Gamma(5)}+2 \frac{t^{9 / 2}}{\Gamma(5.5)}+\frac{t^{5}}{\Gamma(6)}$ \\
3 & $-\frac{t^{4}}{\Gamma(5)}-2 \frac{t^{9 / 2}}{\Gamma(5.5)}-\frac{t^{5}}{\Gamma(6)}$ & $-\frac{t^{11 / 2}}{\Gamma(6.5)}-\frac{t^{6}}{\Gamma(7)}-\frac{t^{13 / 2}}{\Gamma(7.5)}-\frac{t^{7}}{\Gamma(8)}$ \\
$\ldots$ & $\ldots$ & $\ldots$ \\
\hline
\end{tabular}

The ADM method generates the modes as follows:

$$
\begin{aligned}
x_{0} & =\Phi+L^{-1} G=D_{t}^{-2} \delta(t), \\
A_{0} & =x_{0} \\
x_{1} & =-L^{-1} R\left(x_{0}\right)-L^{-1} A_{0}=-\left(D_{t}^{-3 / 2}+D_{t}^{-2}\right) x_{0} \\
& =-\left(\frac{t^{5 / 2}}{\Gamma(7 / 2)}+\frac{t^{3}}{\Gamma(4)}\right) \\
A_{i} & =x_{1}=-\left(\frac{t^{5 / 2}}{\Gamma(7 / 2)}+\frac{t^{3}}{\Gamma(4)}\right), \\
x_{2} & =-L^{-1} R\left(x_{1}\right)-L^{-1} A_{1}=-\left(D_{t}^{-3 / 2}+D_{t}^{-2}\right) x_{1} \\
& =\left(\frac{t^{4}}{\Gamma(5)}+2 \frac{t^{9 / 2}}{\Gamma(11 / 2)}+\frac{t^{5}}{\Gamma(6)}\right) .
\end{aligned}
$$

9.2. Forcing Function as Step Function. Consider a fractional oscillator described by as follows:

$$
D_{t}^{2} x(t)+\frac{c}{m} D_{t}^{1 / 2} x(t)+\frac{k}{m} x(t)=\frac{1}{m} f(t) .
$$

The system is initially stationary (i.e., $x(0)=0$ and $D_{t}^{1}[x(t)]_{t=0}=\dot{x}(0)=0$ are initial conditions). Let this stationary fractional oscillator be subjected to an excitation function $f(t)=F h(t)$, where $h(t)$ is Heaviside's unit step function and $F$ is a constant. By application of ADM (29) (30) and with the help of Figure 3 we obtain the reactionary displacement modes as in the following:

$$
\begin{aligned}
x_{0}(t)= & \frac{1}{m} D_{t}^{-2} f(t)=\frac{F t^{2}}{2 m}, \\
x_{1}(t)= & -\frac{c}{m} D_{t}^{-3 / 2} x_{0}(t)-\frac{k}{m} D_{t}^{-2} x_{0}(t) \\
= & -\frac{c F}{m^{2}} \frac{t^{7 / 2}}{\Gamma(9 / 2)}-\frac{k F}{m^{2}} \frac{t^{4}}{\Gamma(5)}, \\
x_{2}(t)= & -\frac{c}{m} D_{t}^{-3 / 2} x_{1}(t)-\frac{k}{m} D_{t}^{-2} x_{1}(t) \\
= & \frac{c^{2} F}{m^{3}} \frac{t^{5}}{\Gamma(6)}+\frac{2 k c F}{m^{3}} \frac{t^{11 / 2}}{\Gamma(13 / 2)}+\frac{k^{2} F}{m^{3}} \frac{t^{6}}{\Gamma(7)}, \\
x_{3}(t)= & -\frac{c}{m} D_{t}^{-3 / 2} x_{2}(t)-\frac{k}{m} D_{t}^{-2} x_{2}(t), \\
x_{3}(t)= & -\frac{c^{3} F}{m^{4}} \frac{t^{13 / 2}}{\Gamma(15 / 2)}-\frac{3 k c^{2} F}{m^{4}} \frac{t^{7}}{\Gamma(8)} \\
& -\frac{3 k^{2} c F}{m^{4}} \frac{t^{15 / 2}}{\Gamma(17 / 2)}-\frac{k^{3} F}{m^{4}} \frac{t^{8}}{\Gamma(9)} .
\end{aligned}
$$


The total solution is sum of all these component modes to infinity as series in the

$$
\begin{aligned}
x(t)= & x_{0}(t)+x_{1}(t)+x_{2}(t)+x_{3}(t)+\cdots+\cdots, \\
x(t)= & \frac{F}{m} \sum_{r=0}^{\infty} \frac{(-1)^{r}}{r !}\left(\frac{k}{m}\right)^{r} t^{2(r+1)} \\
& \times \sum_{j=0}^{\infty}\left(\frac{-c}{m}\right)^{j} \frac{(j+r) ! t^{3 j / 2}}{j ! \Gamma((3 j / 2)+2 r+3)}, \\
x(t)= & \frac{F}{m} \sum_{r=0}^{\infty} \frac{(-1)^{r}}{r !}\left(\frac{k}{m}\right)^{r} t^{2(r+1)} \\
& \times E_{(3 / 2),(r / 2)+3}^{(r)}\left(\frac{-c}{m} t^{3 / 2}\right),
\end{aligned}
$$

where $E_{\alpha, \beta}(x)$ is two parameter Mittag-Leffler function $[19,21-23]$, and $E_{\alpha, \beta}^{(r)}(x)$ is the derivative of Mittag-Leffler function, $[19,21-23]$ defined as follows:

$$
\begin{array}{r}
E_{\alpha, \beta}^{(r)}(x)=\frac{d^{r}}{d x^{r}} E_{\alpha, \beta}(x)=\sum_{j=0}^{\infty} \frac{(j+r) ! x^{j}}{j ! \Gamma(\alpha j+\alpha r+\beta)} \\
(r=0,1,2,3 \ldots) .
\end{array}
$$

9.3. Explanation of Physical Action Reaction Process vis-àvis $A D M$. The fundamentals of mode decomposition as explained above in case of second-order differential equation, in the presence of fractional order component, may be explained in slightly elaborated way as follows with a mass spring fractional viscous system as

$$
\frac{d^{2}}{d t^{2}} x(t)+a \frac{d^{1 / 2}}{d t^{1 / 2}} x(t)+b x(t)=f(t),
$$

where $a$ is the constant of half-order property and $b$ is the spring stiffness constant for spring. The above equation of motion is for unit mass attached to an ideal spring with half order visco-elastic element [19].

The initial conditions are: $x(0)=0, \dot{x}(0)=0, f(t)=$ $\delta(t)$, for delta function input case. The above equation (67) can be rewritten in terms of external force and opposing internal forces as:

$$
\frac{d^{2}}{d t^{2}} x(t)=f(t)-a \frac{d^{1 / 2}}{d t^{1 / 2}} x(t)-b x(t)
$$

Decomposing the above by modal decomposition, we get the zero order mode as first reaction (immediate) that is: $x_{0}=D_{t}^{-2} f(t)$. This zero order displacement is the reaction without the presence of the spring or any other opposing elements. Due to this zero order, displacement there will be opposing forces appear as $f_{11}=-a D_{t}^{1 / 2} x_{0}$ and $f_{12}=-b x_{0}$. Giving rise to first order displacements as

$$
x_{11}=-D_{t}^{-2} f_{11}=-a D_{t}^{-3 / 2} x_{0}, \quad x_{12}=-D_{t}^{-2} f_{12}=-b D_{t}^{-2} x_{0} .
$$

The overall first modal displacement is: $x_{1}=x_{11}+x_{12}$. From this, the reaction force for second modes is generated as

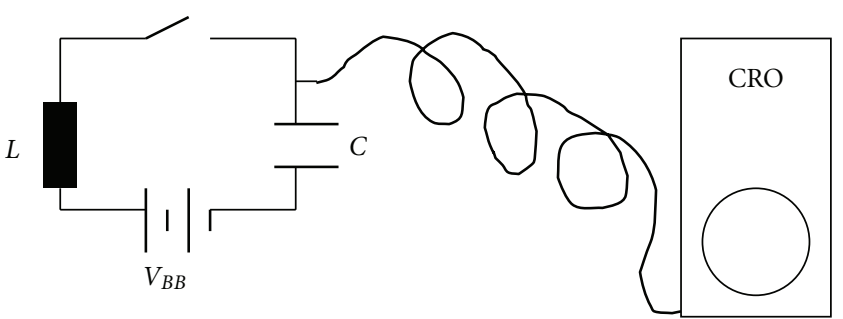

Figure 6: The oscillator circuit (a second-order differential equation), with semi-infinite cable CRO-probe acting as half order element.

$f_{21}=-a D_{t}^{1 / 2} x_{1}$ and $f_{22}=-b x_{1}$. Giving rise to second modal displacement as

$$
x_{21}=-D_{t}^{-2} f_{11}=-a D_{t}^{-3 / 2} x_{1}, \quad x_{22}=-D_{t}^{-2} f_{22}=-b D_{t}^{-2} x_{1} .
$$

Similarly, we can carry on for infinity as this self-similar pattern of reactions generated within the system to external stimulus. The observation is that the half order element adds second force to the ideal spring restoring force as obtained in case of pure second-order pure oscillator (13).

The practical way of explaining the fractional order behavior is by considering a LC-oscillator and then trying to measure the oscillation by CRO probe, which is semi-infinite cable (with losses) acting as half order element [19]. The constant $a$ of the half order element (67) is depending on the distributed loss parameter that is per unit series resistance and per unit shunt capacitance.

The constitutive equation for the circuit is (Figure 6) described in

$$
\begin{gathered}
\frac{1}{C} \int_{0}^{t} i(t) d t+L \frac{d}{d t} i(t)+a \frac{d^{-1 / 2}}{d t^{-1 / 2}} i(t)=V_{B B} h(t), \\
\frac{i(t)}{C}+L \frac{d^{2}}{d t^{2}} i(t)+a \frac{d^{1 / 2}}{d t^{1 / 2}} i(t)=V_{B B} \delta(t) .
\end{gathered}
$$

This is a demonstration of the oscillator with fractional order element, shown in Figure 6. Practically in circuit experiments it is observed that a purely oscillating circuit when connected by shunt to a long CRO-probe, goes to damped oscillationsremoval of probe again giving the oscillations. Use of a very short probe to CRO gives oscillation. This is due to the fact that long CRO probe may act as lossy transmission line, behaving as half-order damping element.

\section{Application of Decomposition Method in RL Formulated Partial Fractional Differential Equations Linear Diffusion Wave Equation and Solution to Impulse Forcing Function}

Diffusion of arbitrary order is studied in detail in [24-26]; an attempt is made to obtain series solution with ADM and the physical explanation of several modes generating a reaction. 
This problem example elaborates that the time evolution of process parameter takes place without the forcing function present, $G=0$; only the effect is due to initial value (in this case is Dirac's delta function as process parameter present at spaceorigin). The fractional time rate of change of the process variable is related to the spatial double derivative of the same as expressed in (72). In (72) the formulation of fractional derivative is of Riemann-Liouvelli (RL) scheme.

Let us consider the problem of fractional time diffusion as

$$
\frac{\partial^{\alpha}}{\partial t^{\alpha}} u(x, t)=\frac{\partial^{2}}{\partial x^{2}} u(x, t) .
$$

With $1<\alpha<2$ and have the initial condition as $u\left(x, 0^{+}\right)=$ $\delta(x)$ and $u_{t}\left(x, 0^{+}\right)=0$. The integer order the highest in the fractional order in case of (72) is $m=2$.

Converting the (72) into Caputo derivative formulation [21-23], we obtain

$$
\begin{aligned}
& { }^{C}\left[\frac{\partial^{\alpha}}{\partial t^{\alpha}} u(x, t)\right]+\frac{t^{-\alpha}}{\Gamma(1-\alpha)} u(x, 0)+\frac{t^{1-\alpha}}{\Gamma(2-\alpha)} \dot{u}(x, 0) \\
& \quad=\frac{\partial^{2}}{\partial x^{2}} u(x, t) .
\end{aligned}
$$

Observation here states that the (72) RL derivative of fractional order when changed to Caputo formulation (73) gives rise to extra source/sink terms of the inverse power function of the independent variables in the constituent equation. In (73) after applying the definition of Caputo derivative [21-23] in the fractional operator we get

$$
\begin{aligned}
D_{t}^{-(2-\alpha)} & {\left[\frac{\partial^{2}}{\partial t^{2}} u(x, t)\right]=\frac{\partial^{2}}{\partial x^{2}} u(x, t) } \\
& -\frac{t^{-\alpha}}{\Gamma(1-\alpha)} u(x, 0)-\frac{t^{1-\alpha}}{\Gamma(2-\alpha)} \dot{u}(x, 0) .
\end{aligned}
$$

Inverting (74), we get

$$
\begin{aligned}
{\left[\frac{\partial^{2}}{\partial t^{2}} u(x, t)\right]=} & D_{t}^{(2-\alpha)}\left[\frac{\partial^{2}}{\partial x^{2}} u(x, t)\right]-\frac{u(x, 0)}{\Gamma(1-\alpha)} D_{t}^{(2-\alpha)}\left[t^{-\alpha}\right] \\
& -\frac{\dot{u}(x, 0)}{\Gamma(2-\alpha)} D_{t}^{(2-\alpha)}\left[t^{1-\alpha}\right] .
\end{aligned}
$$

Applying fractional derivative of the power functions in RHS of (75), we get

$$
\begin{aligned}
{\left[\frac{\partial^{2}}{\partial t^{2}} u(x, t)\right]=} & D_{t}^{(2-\alpha)}\left[\frac{\partial^{2}}{\partial x^{2}} u(x, t)\right]-\frac{u(x, 0)}{\Gamma(1-\alpha)} \\
& \times\left[\frac{\Gamma(-\alpha+1) t^{-\alpha-2+\alpha}}{\Gamma(-\alpha+1-2+\alpha)}\right]-\frac{\dot{u}(x, 0)}{\Gamma(2-\alpha)} \\
& \times\left[\frac{\Gamma(1-\alpha+1) t^{1-\alpha-2+\alpha}}{\Gamma(1-\alpha+1-2+\alpha)}\right] .
\end{aligned}
$$

Simplifying (76), we have

$$
\begin{aligned}
{\left[\frac{\partial^{2}}{\partial t^{2}} u(x, t)\right]=} & D_{t}^{(2-\alpha)}\left[\frac{\partial^{2}}{\partial x^{2}} u(x, t)\right] \\
& -u(x, 0)\left[\frac{t^{-2 \alpha}}{\Gamma(-1)}\right]-\dot{u}(x, 0)\left[\frac{t^{-1}}{\Gamma(0)}\right] .
\end{aligned}
$$

The last two terms of (77) gives zero; since reciprocal Gamma function is zero at values zero and negative integer points giving the modified diffusion equation as

$$
\left[\frac{\partial^{2}}{\partial t^{2}} u(x, t)\right]=D_{t}^{(2-\alpha)}\left[\frac{\partial^{2}}{\partial x^{2}} u(x, t)\right] .
$$

Taking the (space) Fourier Transform of (78), we get

$$
\frac{\partial^{2}}{\partial t^{2}} \bar{u}(k, t)=-k^{2} D_{t}^{(2-\alpha)} \bar{u}(k, t) .
$$

With transformed initial condition as $\bar{u}(k, 0)=(1 / \sqrt{2 \pi})$; and $\bar{u}_{t}(k, 0)=0$, where $\bar{u}(k, t)=(1 / \sqrt{2 \pi}) \int_{-\infty}^{+\infty} e^{i k x} u(x, t) d x, k \in$ $\mathfrak{R}$ is the spatial Fourier Transform definition. The parameter $k$ is "wave-vector". The system of equations (78) has been transformed to (79) so we solve for $\bar{u}(k, t)$ and write with $\operatorname{ADM}(29)$ the solution as

$$
\begin{aligned}
\bar{u}(k, t) & =\Phi+L^{-1} G-L^{-1} R(u)-L^{-1} N(u), \\
\bar{u}(k, t) & =\Phi+L^{-1}\left[D_{t}^{2-\alpha} \bar{u}(k, t)\right], \\
\bar{u}(k, t) & =\bar{u}(k, 0)+t \bar{u}_{t}(k, 0)-k^{2} D_{t}^{-2}\left[D_{t}^{2-\alpha} \bar{u}(k, t)\right] \\
& =\bar{u}(k, 0)-k^{2} D_{t}^{-2}\left[D_{t}^{2-\alpha} \bar{u}(k, t)\right] .
\end{aligned}
$$

In (80) the $L^{-1} D_{t}^{-2}, G=R=0, N(u)=\bar{u}(k, t)$, is the linear.

Therefore, $\bar{u}_{0}=\Phi=\bar{u}_{0}(k, 0)$ and for $n \geq 1 ; \bar{u}_{n}=$ $-L^{-1} D_{t}^{2-\alpha} \bar{u}_{n-1}$; Following the ADM, (29) we get $\bar{u}(k, t)=$ $\sum_{n=0}^{\infty} \bar{u}_{n}(k, t)$, where the components are listed in

$$
\begin{gathered}
\bar{u}_{0}=\frac{1}{\sqrt{2 \pi}}, \\
A_{0}=\bar{u}_{0},
\end{gathered}
$$

$$
\bar{u}_{1}=-L^{-1} D_{t}^{2-\alpha}\left[A_{0}\right]-k^{2} D_{t}^{-2}\left[D_{t}^{2-\alpha} \bar{u}_{0}\right]=-\frac{k^{2}}{\sqrt{2 \pi}}\left(\frac{t^{\alpha}}{\Gamma(\alpha+1)}\right),
$$$$
A_{1}=\bar{u}_{1},
$$$$
\bar{u}_{2}=-k^{2} D_{t}^{-2}\left[D_{t}^{2-\alpha} \bar{u}_{1}\right]=\frac{k^{4}}{\sqrt{2 \pi}}\left(\frac{t^{2 \alpha}}{\Gamma(2 \alpha+1)}\right),
$$$$
A_{2}=\bar{u}_{2},
$$$$
\bar{u}_{3}=-k^{2} D_{t}^{-2}\left[D_{t}^{2-\alpha} \overline{\mathcal{u}}_{2}\right]=-\frac{k^{6}}{\sqrt{2 \pi}}\left(\frac{t^{3 \alpha}}{\Gamma(3 \alpha+1)}\right),
$$

$$
\bar{u}(k, t)=\frac{1}{\sqrt{2 \pi}}\left[1-\frac{k^{2} t^{\alpha}}{\Gamma(\alpha+1)}+\frac{k^{4} t^{2 \alpha}}{\Gamma(2 \alpha+1)}-\frac{k^{6} t^{3 \alpha}}{\Gamma(3 \alpha+1)}+\cdots\right] .
$$


Therefore,

$$
\bar{u}(k, t)=\frac{1}{\sqrt{2 \pi}} \sum_{n=0}^{\infty} \frac{\left(k^{2}\right)^{n} t^{n \alpha}}{\Gamma(n \alpha+1)}=\frac{1}{\sqrt{2 \pi}} E_{\alpha}\left(-k^{2} t^{\alpha}\right) .
$$

Equation (83) is series solution of (79) in space-Fourier Transformed system, in terms of Mittag-Leffler function. Here, is one parameter Mittag-Leffler function $E_{\alpha}\left(-k^{2} t^{\alpha}\right)$. Taking the Inverse Fourier Transform of (83), we get solution to (72) with impulse excitation as

$$
u(x, t)=\frac{1}{2} t^{-\alpha / 2} M_{\alpha / 2}\left(\frac{|x|}{t^{\alpha / 2}}\right) ; \quad \text { for }-\infty<x<+\infty, t \geq 0,
$$

where Inverse Fourier transform is: $u(x, t)=(1 /$ $\sqrt{2 \pi}) \int_{-\infty}^{+\infty} e^{-i k x} \bar{u}(k, t) d k, x \in \Re$.

The $M_{\alpha / 2}$ is special case of Wright function [21] defined as in

$$
\begin{gathered}
M_{\gamma}(|z|)=\sum_{n=0}^{\infty} \frac{(-|z|)^{n}}{n ! \Gamma(-n \gamma+\{1-\gamma\})}, \quad \text { where } 0<\gamma<1, \\
M_{\alpha / 2}\left(\frac{|x|}{t^{\alpha / 2}}\right)=\sum_{n=0}^{\infty} \frac{\left(-|x| / t^{\alpha / 2}\right)^{n}}{n ! \Gamma[-(n \alpha / 2)+(1-(\alpha / 2))]}, \\
\text { where } 0<\frac{\alpha}{2}<1 .
\end{gathered}
$$

\section{Generalization of Fractional-Order Leading Terms in Differential Equations Formulated with Riemann-Liouvelli and Caputo Definitions and Use of Integer-Order Initial/Boundary Conditions with Decomposition Method}

In this section merger of the two classical definitions of Fractional Derivatives with decomposition, the technique is demonstrated; where only integer order initial/boundary conditions will be employed to get to the modal solutions in decomposition method. This generalization and unification is important as to eliminate the need of much difficult fractional order initial states required classically by $\mathrm{RL}$ formulation of FDE.

11.1. Decomposition of Caputo Derivative in Fractional Differential Equations. Let the linear part of the equation $\mathrm{Lu}+$ $R u+N u=G$ be of Caputo Fractional Derivative represented as $L={ }^{C} D_{t}^{\alpha}$, which is composed of integer order derivative of function followed by fractional integration. That is if an integer $m$ is just greater than fraction $\alpha>0$; that is $(m-1)<$ $\alpha<m$, then; Caputo operator is: ${ }^{C} D_{t}^{\alpha} \equiv D_{t}^{-(m-\alpha)} D_{t}^{m} \cdot[19,21-$ 23]. This gives the differential equation system as

$$
D_{t}^{-(m-\alpha)} D_{t}^{m}(u)=G-R(u)-N(u) .
$$

Inverting this, we get

$$
D_{t}^{m}(u)=D_{t}^{(m-\alpha)} G-D_{t}^{(m-\alpha)} R(u)-D_{t}^{(m-\alpha)} N(u) .
$$

The solution is

$$
u(t)=\Phi+L^{-1} D_{t}^{m-\alpha} G-L^{-1} D_{t}^{m-\alpha} R(u)-L^{-1} D_{t}^{m-\alpha} N(u)
$$

where $L^{-1}=D_{t}^{-m}$; we have used complementation property that is $D^{m} D^{-m}=I$.

The $\Phi$ is solution to integer order. Homogeneous condition of $D_{t}^{m} u(t)=0$ is the same as in the case of integer order general differential equation solution described previously. Due to this fact, researchers like to formulate with Caputo derivative [27-30].

11.2. Riemann-Liouvelli ( $R L)$ Derivative and Its Decomposition for Solving Fractional Differential Equation with Integer Order Initial Condition. The RL derivative operator is $D_{t}^{\alpha} \equiv D_{t}^{m} D_{t}^{-(m-\alpha)}$. That is, the function is first fractionally integrated and then differentiated by integer order, which is just greater than the fractional order. [19, 21-23]. In this solution, the homogeneous equation formed by RL operator requires fractional initial states; [19, 21-23] though sometimes difficult to interpret physically. However, one can relate these fractional initial states to physical quantities provided the laws of physics are known [19] (As Ohm's Law, Stress-Strain relations and flow-pressure relations etc.) Here in this paper, it is demonstrated that decomposing fractional derivative with RL definition by transforming to Caputo expression first, then applying decomposition rules, one can solve the fractional differential equations with RL formulations and with integer order initial states.

Generally, the Caputo and RL definitions of fractional derivatives are not equal, but are equated by initial conditions as [21-23]

$$
\begin{aligned}
{\left[D_{t}^{\alpha} f(t)\right]_{\mathrm{RL}}=} & { }^{C} D_{t}^{\alpha} f(t)+\sum_{k=0}^{m-1} \frac{t^{k-\alpha}}{\Gamma(k-\alpha+1)} f^{(k)}\left(0^{+}\right), \\
& \text {where }(m-1)<\alpha<m, m \in \mathrm{N}, t>0 .
\end{aligned}
$$

Let the linear part $L$ be of RL derivative type. Then the formulation with definition of RL derivative [19, 21-23] gives the system as: $D_{t}^{m} D_{t}^{-(m-\alpha)}(u)=G-R(u)-N(u)$. In the expression with RL, we change to Caputo and relate with RL-Caputo relation [21-23] and get

$$
{ }^{C} D_{t}^{\alpha}(u)=G-R(u)-N(u)-\sum_{k=0}^{m-1} \frac{t^{k-\alpha}}{\Gamma(k-\alpha+1)} u^{(k)}\left(0^{+}\right) .
$$

Here the RL differential equation is changed to Caputo formulation. This is equivalent to original differential equation, but with extra power series term with integer order initial conditions appearing as extra source/sink term. Let us 
follow the decomposition method, as obtained for Caputo formulation in Section 11.1, thereby giving the solution as

$$
\begin{aligned}
u(t)= & \Phi+L^{-1} D_{t}^{m-\alpha} G-L^{-1} D_{t}^{m-\alpha} R(u)-L^{-1} D_{t}^{m-\alpha} N(u) \\
& -L^{-1}\left\{D_{t}^{m-\alpha} \sum_{k=0}^{m-1} \frac{t^{k-\alpha}}{\Gamma(k-\alpha+1)} u^{(k)}\left(0^{+}\right)\right\}
\end{aligned}
$$

Let us examine the bracketed term of the RHS of the source sink term.

$$
\begin{aligned}
& D_{t}^{m-\alpha} \sum_{k=0}^{m-1} \frac{t^{k-\alpha}}{\Gamma(k-\alpha+1)} u^{(k)}\left(0^{+}\right) \\
& \quad=u^{(k)}\left(0^{+}\right) \sum_{k=0}^{m-1} \frac{\Gamma(k-\alpha+1) t^{k-\alpha-m+\alpha}}{\Gamma(k-\alpha+1) \Gamma(k-\alpha+1-m+\alpha)} \\
& \quad=u^{(k)}\left(0^{+}\right) \sum_{k=0}^{m-1} \frac{t^{k-m}}{\Gamma(k-m+1)} .
\end{aligned}
$$

The expression above contains reciprocal of Gamma function at negative integer points and zero point, the values of which are zero. The reciprocal of Gamma functions $\Gamma(-(m-1)), \Gamma(-(m-2)), \ldots, \Gamma(0)$ are zeros. This reciprocal Gamma function is multiplied by powers of $t$ at $t \rightarrow 0^{+}=$ $\varepsilon$; as $\varepsilon^{-m}, \varepsilon^{1-m}, \varepsilon^{2-m}, \ldots \varepsilon^{-1}$. Therefore, at $t \rightarrow 0^{+}$, the inverted source/sink extra term be collapsed to zero, that is, $D_{t}^{m-\alpha} \sum_{k=0}^{m-1}\left(t^{k-\alpha} / \Gamma(k-\alpha+1)\right) u^{(k)}\left(0^{+}\right)=0$.

The fractional differentiation of a power function is given by $D^{\mu} t^{\lambda}=\Gamma(\lambda+1) t^{\lambda-\mu} / \Gamma(\lambda+1-\mu)$, Euler's rule of generalized differ-integration $[19,21-23]$, where $\mu \in \mathfrak{R}^{+}$, with $\lambda>-1$ Let us take monomial of type say $x^{n}$, with $n$ as integer. We differentiate this with integer order $m$ such that $(m-n)=1,2,3, \ldots$. Then in the integer order calculus $D_{x}^{m} x^{n}=0$. Say a square function $x^{2}$ differentiated thrice, four times, and so on will give zero. Same is the observation for $D_{t}^{(m-\alpha)} t^{k-\alpha}$, returns zero since differential order (fractional) minus the power order that is $(m-\alpha)-(k-\alpha)=m-k$, is $m,(m-1),(m-2), \ldots, 1$, for $k=0,1,2,3, \ldots(m-1)$. This is new observation not used elsewhere earlier in RL fractional calculus context. This new observation and its application is now useful for solving FDE with RL formulation by decomposition technique where the extra source sink term appearing in FDE (changing from RL to Caputo) collapses to zero thus giving ease and uniformity in the two definitions of fractional calculus.

The above argument suggests that with $\mathrm{RL}$ derivative formulations too one can have solution in ADM approach to solve fractional differential equation, with the help of integer order initial/boundary condition.

Therefore, the solution of General Fractional Order Differential Equation where the leading terms are of Fractional Derivative of Caputo or RL type is

$$
\begin{aligned}
u(t)= & \Phi+L^{-1} D_{t}^{m-\alpha} G-L^{-1} D_{t}^{m-\alpha} R(u) \\
& -L^{-1} D_{t}^{m-\alpha} N(u),
\end{aligned}
$$

where $\Phi$ comes from integer order initial/boundary conditions. This unifies the two definitions of Caputo/RL to solve FDE with only integer order initial states.

\section{Application of Decomposition Method in RL Formulated Fractional Differential Equations (Nonlinear) and Its Solution}

So far, we considered linear systems and reasoned out physically the decomposition and the action-reaction concepts to solve the differential equation systems, by ADM. The nonlinear part $N(u)$ in the earlier cases were linear in nature and thus the Adomian Polynomials for each mode were same $\left(A_{n}=u_{n}\right)$ for $n \geq 1$, for obtaining the subsequent parallel modes and thereby the solution. The non-linear part is described giving different Adomian Polynomials for the different modes to get solution of non-linear systems.

Consider RL formulated Fractional differential equation with non-linearity as in

$$
\frac{d^{\alpha} y}{d t^{\alpha}}=(1-y)^{4}, \quad \text { with } 0<\alpha<1, y\left(0^{+}\right)=0 .
$$

The nearest integer in this case is, for the fractional order, $\alpha$. The invert operator, $L^{-1}=D_{t}^{-1}$ and $\Phi=C=0$ the solution is thus; as in (72)-(78) is

$$
y(t)=\sum_{n=0}^{\infty} y_{n}=\Phi+L^{-1} D_{t}^{1-\alpha} \sum_{n=0}^{\infty} A_{n}=D_{t}^{-1}\left[D^{1-\alpha} \sum_{k=0}^{\infty} A_{n}\right] .
$$

The source/sink term that appears in the RL to Caputo change in (94) is $-\left(t^{-\alpha} / \Gamma(1-\alpha)\right) y\left(0^{+}\right)$. Taking $D_{t}^{m-\alpha}$ of this source/sink term gives

$$
-\frac{y\left(0^{+}\right) \Gamma(-\alpha+1) t^{-\alpha-m+\alpha}}{\Gamma(1-\alpha) \Gamma(-\alpha+1-m+\alpha)}=-\frac{y\left(0^{+}\right) t^{-1}}{\Gamma(0)}=0
$$

therefore, the decomposed solution of (94) is (96).

The non-linearity $N(y)=(1-y)^{4}=\sum_{n=0}^{\infty} A_{n}$, and to find the Adomian Polynomials following are the steps (29) and (30).

The iterations are listed in

$$
\begin{gathered}
y_{0}=0, \quad A_{0}=\left(1-y_{0}\right)^{4}=1, \\
y_{1}(t)=D_{t}^{-1} D_{t}^{1-\alpha}\left[A_{0}\right]=D_{t}^{-1} D_{t}^{1-\alpha}(1)=\frac{t^{\alpha}}{\Gamma(\alpha+1)}, \\
A_{1}=y_{1} N^{\prime}\left(y_{0}\right), \\
N^{\prime}\left(y_{0}\right)=\frac{d}{d y_{0}}\left(1-y_{0}\right)^{4}=-4\left(1-y_{0}\right)^{3}=-4, \\
A_{1}=y_{1}(-4)=-\frac{4 t^{\alpha}}{\Gamma(\alpha+1)} .
\end{gathered}
$$


From this, we obtain the next term of solution as

$$
y_{2}(t)=D_{t}^{-1} D_{t}^{1-\alpha}\left[A_{1}\right]=D_{t}^{-1} D_{t}^{1-\alpha}\left[\frac{-4 t^{\alpha}}{\Gamma(\alpha+1)}\right]=\frac{-4 t^{2 \alpha}}{\Gamma(2 \alpha+1)}
$$

Next step is to obtain $A_{2}$ as

$$
\begin{gathered}
A_{2}=y_{2} N^{\prime}\left(y_{0}\right)+\frac{y_{1}^{2}}{2 !} N^{\prime \prime}\left(y_{0}\right), \\
N^{\prime \prime}\left(y_{0}\right)=\frac{d^{2}}{d y_{0}^{2}}\left(1-y_{0}\right)^{4}=12\left(1-y_{0}\right)^{2}=12, \\
N^{\prime}\left(y_{0}\right)=-4, \\
A_{2}=\left(\frac{-4 t^{2 \alpha}}{\Gamma(2 \alpha+1)}\right)(-4)+\frac{12}{2}\left(\frac{t^{\alpha}}{\Gamma(\alpha+1)}\right)^{2}, \\
=\frac{6 \Gamma(2 \alpha+1)-16[\Gamma(\alpha+1)]^{2}}{\Gamma(2 \alpha+1)[\Gamma(\alpha+1)]^{2}} t^{2 \alpha} .
\end{gathered}
$$

From above (99) we obtain

$$
y_{3}(t)=D_{t}^{-1} D_{t}^{1-\alpha}\left[A_{2}\right]=\frac{6 \Gamma(2 \alpha+1)-16[\Gamma(\alpha+1)]^{2}}{\Gamma(3 \alpha+1)[\Gamma(\alpha+1)]^{2}} t^{3 \alpha} .
$$

The series form solution to (94) is

$$
\begin{aligned}
y(t)= & \frac{1}{\Gamma(\alpha+1)} t^{\alpha}-\frac{4}{\Gamma(2 \alpha+1)} t^{2 \alpha} \\
& +\frac{6 \Gamma(2 \alpha+1)-16[\Gamma(\alpha+1)]^{2}}{\Gamma(3 \alpha+1)[\Gamma(\alpha+1)]^{2}} t^{3 \alpha}+\cdots
\end{aligned}
$$

\section{Application of Decomposition Method in RL Formulated Partial Fractional Differential Equations Nonlinear Diffusion-Wave Equation and Solution}

Consider the Non-Linear equation (102), formulated by RL fractional differential operator

$$
\frac{\partial^{\alpha}}{\partial t^{\alpha}} u(x, t)=\gamma^{2} \frac{\partial^{2}}{\partial x^{2}} u(x, t)-c^{2} u(x, t)+\sigma[u(x, t)]^{3}
$$

with $1<\alpha \leq 2$ and $u(x, 0)=\varepsilon \cos k x$, and the first time derivative $u_{t}(x, 0)=0$ as initial condition.

The argument about vanishing extra source/sink term while RL is changed to Caputo formulation in this case is the same as in the previous problem (72)-(78).

In this case, $m=2$, that is integer number just greater than $\alpha$ in the order of fractional derivative of (102). The nonlinear part is $N(u)=c^{2} u-\sigma u^{3}=\sum_{n=0}^{\infty} A_{n}$, and we simplify the (102) in easier notation as $D_{t}^{\alpha} u=\gamma^{2} u_{x x}-c^{2} u+\sigma u^{3}$; where $u_{x x} \equiv\left(\partial^{2} / \partial x^{2}\right) u(x, t)$. From $(72)-(78)$ we write the solution of (102) as

$$
\begin{aligned}
u(x, t)= & \Phi+\gamma^{2} D_{t}^{-2} D_{t}^{2-\alpha}\left[u_{x x}\right]-D_{t}^{-2} D_{t}^{2-\alpha} N(u), \\
u(x, t)= & u(x, 0)+t u_{t}(x, 0)+\gamma^{2} D_{t}^{-2} D_{t}^{2-\alpha}\left[u_{x x}\right] \\
& -D_{t}^{-2} D_{t}^{2-\alpha} \sum_{n=0}^{\infty} A_{n} .
\end{aligned}
$$

The iteration follows as shown in steps

$$
\begin{aligned}
u_{0} & =\Phi=u(x, 0)=\varepsilon \cos k x, \\
A_{0} & =c^{2} u_{0}-\sigma u_{0}^{3}=c^{2} \varepsilon \cos k x-\sigma \varepsilon^{3} \cos ^{3} k x, \\
u_{1} & =\gamma^{2} D_{t}^{-2} D_{t}^{2-\alpha} u_{0_{(x x)}}-D_{t}^{-2} D_{t}^{2-\alpha} A_{0}, \\
u_{0_{(x x)}} & =\frac{\partial^{2} u_{0}}{\partial x^{2}}=-\varepsilon k^{2} \cos k x,
\end{aligned}
$$

$D_{t}^{-2} u_{0_{(x x)}}=\iint_{0}^{t} u_{0_{(x x)}} d t d t=-\frac{\varepsilon k^{2} t^{2}}{2} \cos k x$

$$
\begin{aligned}
& D_{t}^{-2} A_{0}=\iint_{0}^{t} A_{0} d t d t=\frac{t^{2} c^{2}}{2} \varepsilon \cos k x-\frac{\sigma \varepsilon^{3} t^{2}}{2} \cos ^{3} k x, \\
& u_{1}=-\frac{\gamma^{2} \varepsilon k^{2} \cos k x}{\Gamma(\alpha+1)} t^{\alpha}-\frac{\left(c^{2} \varepsilon \cos k x-\sigma \varepsilon^{3} \cos ^{3} k x\right)}{\Gamma(\alpha+1)} t^{\alpha} \text {, } \\
& A_{1}=u_{1}\left(c^{2}-3 \sigma u_{0}^{2}\right) \text {, } \\
& u_{2}=\gamma^{2} D_{t}^{-2} D_{t}^{2-\alpha} u_{1_{(x x)}}-D_{t}^{-2} D_{t}^{2-\alpha} A_{1}, \\
& u_{1_{(x x)}}=\frac{\partial^{2} u_{1}}{\partial x^{2}} \\
& u_{2}=\frac{\varepsilon \gamma^{4} k^{4} \cos k x}{\Gamma(2 \alpha+1)} t^{2 \alpha}-\left[\frac{\gamma^{2}}{\Gamma(2 \alpha+1)}\right] \\
& \times\left[-c^{2} \varepsilon k^{2} \cos k x+\frac{3 k^{2} \sigma \varepsilon^{3}}{4} \cos k x+\frac{9 k^{2} \sigma \varepsilon^{3}}{4} \cos ^{3} k x\right] \\
& \times t^{2 \alpha}+\frac{c^{2} \varepsilon \gamma^{2} k^{2} \cos k x}{\Gamma(2 \alpha+1)} t^{2 \alpha} \\
& +\frac{c^{2}\left(c^{2} \varepsilon \cos k x-\sigma \varepsilon^{3} \cos ^{3} k x\right)}{\Gamma(2 \alpha+1)} t^{2 \alpha} \\
& -\frac{3 \sigma \varepsilon^{3} \gamma^{2} k^{2} \cos ^{3} k x}{\Gamma(2 \alpha+1)} t^{2 \alpha} \\
& -\frac{3 \sigma \varepsilon^{2} \cos ^{2} k x\left(c^{2} \varepsilon \cos k x-\sigma \varepsilon^{3} \cos ^{3} k x\right)}{\Gamma(2 \alpha+1)} t^{2 \alpha} .
\end{aligned}
$$

Therefore, the approximate (three term) solution to (102) is $u(x, t) \approx u_{0}+u_{1}+u_{2}=\sum_{n=0}^{2} u_{n}$. 


\section{Decomposition Method for Generalized Equation of Motion}

Consider the equation of motion (105) a non-linear one; where two bodies of mass $m_{1}$ and $m_{2}$ collide and the variable $x(t)$ denotes indentation with respect to time [20]. The contact force is Hertz force given by $f=K x^{n}$. For spherical particles, this index of non-linearity is $n=3 / 2$. The parameter $K$ is depending on the contact properties of material

$$
\frac{m_{1} m_{2}}{2\left(m_{1}+m_{2}\right)} \frac{d^{2} x}{d t^{2}}+K(n+1) x^{n}=0
$$

We consider the (105) in simple way as

$$
\frac{d^{2} x}{d t^{2}}=-a x^{3 / 2}, \quad \text { where } n=\frac{3}{2}, \quad a=\frac{5\left(m_{1}+m_{2}\right) K}{m_{1} m_{2}}
$$

with $x(0)=0$ and $D_{t}^{1}[x(t)]_{t=0}=\dot{x}(0)=u$ as the initial conditions. The (106) has $G=0, R=0, L^{-1}=D_{t}^{-2}$, $N(x)=a x^{3 / 2}$. The ADM for (106) gives set as

$$
\begin{aligned}
\Phi & =x(0)+t \dot{x}(0)=u t, \\
x_{0} & =\Phi+L^{-1} G=u t \\
A_{0} & =N\left(x_{0}\right)=a x_{0}^{3 / 2}=a u^{3 / 2} t^{3 / 2}, \\
x_{1} & =-L^{-1} R\left(x_{0}\right)-L^{-1}\left(A_{0}\right) \\
& =-D_{t}^{-2}\left[a u^{3 / 2} t^{3 / 2}\right]=-\frac{4}{35} a u^{3 / 2} t^{7 / 2}, \\
A_{1} & =x_{1} N^{\prime}\left(x_{0}\right)=\frac{3}{2} a x_{0}{ }^{1 / 2} x_{1}=-\frac{12}{70} a^{2} u^{2} t^{4}, \\
x_{2} & =-L^{-1} R\left(x_{1}\right)-L^{-1}\left(A_{1}\right) \\
& =-D_{t}^{-2}\left[-\frac{12}{70} a^{2} u^{2} t^{4}\right]=\frac{12}{210} a^{2} u^{2} t^{6}
\end{aligned}
$$

and so on.

The solution, with decomposed modes of displacements as series, to (105) and (106) is

$$
x(t)=u t-\frac{4 a u^{3 / 2}}{35} t^{7 / 2}+\frac{12 a^{2} u^{2}}{210} t^{6}+\cdots
$$

For completion sake let $n=0$, in, (105) and (106). This makes $N\left(x_{0}\right)=a=A_{0}$, and $N^{\prime}(x)=0$, therefore $A_{1}=0=$ $A_{2}=\cdots=A_{\infty}$.

Here, $x_{0}=\Phi+L^{-1} G=u t$ and $x_{1}=-L^{-1} A_{0}=$ $-D_{t}^{-2}(a)=-(1 / 2) a t^{2}$, with higher modes all zero that is $x_{2}=x_{3}=\cdots=x_{\infty}=0$. The solution is just sum of zeroth and first mode of reaction that is $x(t)=u t-(1 / 2) a t^{2}$; these are Newton's familiar laws of motion.

For, $n=1$ (105) becomes oscillatory system (mass spring) described earlier.

\section{Decomposition Method for Delayed Differential Equation System}

In this concluding section, application of mode decomposition in delay differential equation is demonstrated. A delayed differential equation system is

$$
L(u)+R(u)+N(u)=G+f(x, u(x), u(g(x))) .
$$

Consider a nonlinear delayed differential equation system as

$$
\frac{d y}{d x}=1-2 y^{2}\left(\frac{x}{2}\right)
$$

with $y(0)=0$, as initial condition.

Here in (110) $G=1, R=0, L^{-1}=D_{x}^{-1}, N(y)=$ $2 y^{2}(x / 2)$. Applying ADM, we get the set as mentioned in

$$
\begin{aligned}
y_{0}(x) & =\Phi+L^{-1} G=D_{x}^{-1}[1]=x, \\
y_{0}\left(\frac{x}{2}\right) & =\frac{x}{2}, \\
A_{0} & =N\left(y_{0}\right)=2 y_{0}^{2}\left(\frac{x}{2}\right)=-2\left(\frac{x}{2}\right)^{2}=-\frac{1}{2} x^{2}, \\
y_{1}(x) & =-L^{-1}\left(A_{0}\right)=-D_{x}^{-1}\left(\frac{x^{2}}{2}\right)=-\frac{x^{3}}{6}, \\
y_{1}\left(\frac{x}{2}\right) & =-\frac{(x / 2)^{3}}{6}, \\
N^{\prime}\left(y_{0}\right) & =4 y_{0}\left(\frac{x}{2}\right), \quad N^{\prime \prime}\left(y_{0}\right)=4, \\
A_{1} & =y_{1} N^{\prime}\left(y_{0}\right)=y_{1}\left(\frac{x}{2}\right) 4 y_{0}\left(\frac{x}{2}\right) \\
y_{3}(x) & =-L^{-1}\left(A_{2}\right)=-D_{x}^{-1}\left(A_{2}\right)=\frac{x^{7}}{5040} . \\
A_{2} & =y_{2} N^{\prime}\left(y_{0}\right)+\frac{1}{2 !} y_{1}^{2} N^{\prime \prime}\left(y_{0}\right) \\
y_{2}\left(\frac{x}{2}\right) & =\frac{(x / 2)^{5}}{120}, \times 4\left(\frac{x}{2}\right)=-\frac{x^{4}}{24}, \\
y_{2}(x) & =-L^{-1}\left(A_{1}\right)=-D_{x}^{-1}\left(-\frac{x^{4}}{24}\right)=\frac{x^{5}}{120}, \\
& =y_{2}^{6},
\end{aligned}
$$

The solution to $(110)$ is

$$
y(x)=x-\frac{x^{3}}{6}+\frac{x^{5}}{120}-\frac{x^{7}}{5040}+\cdots=\sin x .
$$

This is exact solution to (110) as the RHS of (110) is

$$
1-2 y^{2}\left(\frac{x}{2}\right)=1-2 \sin ^{2}\left(\frac{x}{2}\right)=\cos x=\frac{d(\sin x)}{d x}
$$




\section{Conclusions}

The Decomposition Method of Mathematics of Linear Analysis, as demonstrated, is a generalization of the physical law of nature. That is, the process reacts in opposite way to thwart any changes in the process variable. The Decomposition Method as explained for physical systems gives insight into microscale reactions, to the external or internal stimulus-as to oppose the changes, thereby generating infinite (or finite) modes of reactions, the sum of which gives the total system behavior. In the fractional differential equation (FDE) by RL method, it is found that there is no need to worry about the fractional initial states; instead, one can use integer order initial states (the conventional ones) to arrive at solution of FDE. This new finding too is highlighted in this paper along with several other problems to give physical insight to the solution of extraordinary differential equation systems. This way one gets insight to Physics of General Differential Equation Systems and its solution by Physical Principle and equivalent mathematical decomposition method. This facilitates ease in modeling to get approximate analytic behavior of General Dynamic System involving Extra Ordinary Differential Equations.

\section{Acknowledgment}

This work is dedicated to my blind father Soumendra Kumar Das, who left us forever in July, 2009.

\section{References}

[1] G. Adomian, Nonlinear Stochastic System Theory and Application to Physics, Kluwer Academic Publishers, Dordrecht, The Netherlands, 1989.

[2] G. Adomian, Solving Frontier Problems of Physics: The Decomposition Method, Kluwer Academic Publishers, Boston, Mass, USA, 1994.

[3] G. Adomian, "An analytical solution of the stochastic NavierStokes system," Foundations of Physics, vol. 21, no. 7, pp. 831843, 1991.

[4] G. Adomian and R. Rach, "Linear and nonlinear Schrödinger equations," Foundations of Physics, vol. 21, no. 8, pp. 983-991, 1991.

[5] G. Adomian, "Solution of physical problems by decomposition," Computers and Mathematics with Applications, vol. 27, no. 9-10, pp. 145-154, 1994.

[6] G. Adomian, "Solutions of nonlinear PDE," Applied Mathematics Letters, vol. 11, no. 3, pp. 121-123, 1998.

[7] K. Abbaoui and Y. Cherruault, "The decomposition method applied to the Cauchy problems," Kybernetes, vol. 28, no. 1, pp. 68-74, 1999.

[8] D. Kaya and A. Yokus, "A numerical comparison of partial solutions in the decomposition method for linear and nonlinear partial differential equations," Mathematics and Computers in Simulation, vol. 60, no. 6, pp. 507-512, 2002.

[9] D. Kaya and S. M. El-Sayed, "On a generalized fifth order KdV equations,” Physics Letters A, vol. 310, no. 1, pp. 44-51, 2003.
[10] D. Kaya and S. M. El-Sayed, "An application of the decomposition method for the generalized KdV and RLW equations," Chaos, Solitons and Fractals, vol. 17, no. 5, pp. 869-877, 2003.

[11] D. Kaya, "An explicit and numerical solutions of some fifthorder KdV equation by decomposition method," Applied Mathematics and Computation, vol. 144, no. 2-3, pp. 353-363, 2003.

[12] D. Kaya, "A numerical simulation of solitary-wave solutions of the generalized regularized long-wave equation," Applied Mathematics and Computation, vol. 149, no. 3, pp. 833-841, 2004.

[13] A. J. George and A. Chakrabarti, "The Adomian method applied to some extra ordinary differential equations," Applied Mathematics Letters, vol. 8, no. 3, pp. 91-97, 1995.

[14] H. L. Arora and F. I. Abdelwahid, "Solution of non-linear order differential equations via Adomian decomposition method," Applied Mathematics Letters, vol. 6, no. 1, pp. 21-23, 1993.

[15] N. T. Shawagfeh, "The decomposition method for fractional differential equations," Journal of Fractional Calculus, vol. 16, pp. 27-33, 1999.

[16] Y. Cherruault, "Convergence of Adomian's method," Kybernetes, vol. 18, pp. 31-38, 1989.

[17] K. Abbaoui and Y. Cherruault, "Convergence of Adomian's method applied to differential equations," Computers and Mathematics with Applications, vol. 28, no. 5, pp. 103-109, 1994.

[18] K. Abbaoui and Y. Cherruault, "New ideas for proving convergence of decomposition methods," Computers and Mathematics with Applications, vol. 29, no. 7, pp. 103-108, 1995.

[19] S. Das, Functional Fractional Calculus for System Identifications and Controls, Springer, Berlin, Germany, 2007.

[20] H. M. Lankarani and P. E. Nikravesh, "A contact force model with hysterisis damping for impact analysis of multibody system," Journal of Mechanical Design, vol. 112, pp. 369-376, 1990.

[21] I. Podlubny, Fractional Differential Equations, Academic Press, San Diego, Calif, USA, 1999.

[22] K. B. Oldham and J. Spanier, The Fractional Calculus, Academic Press, New York, NY, USA, 1974.

[23] K. S. Miller and B. Ross, An Introduction to Fractional Calculus and Fractional Differential Equations, John Willey \& Sons, New York, NY, USA, 1993.

[24] O. P. Agrawal, "Solution for a fractional diffusion-wave equation defined in a bounded domain," Nonlinear Dynamics, vol. 29, no. 1-4, pp. 145-155, 2002.

[25] R. Metzler and J. Klafter, “The random walk's guide to anomalous diffusion: a fractional dynamics approach," Physics Report, vol. 339, no. 1, pp. 1-77, 2000.

[26] W. R. Schneider and W. Wyss, "Fractional diffusion and wave equations," Journal of Mathematical Physics, vol. 30, no. 1, pp. 134-144, 1989.

[27] S. Saha-Ray and R. K. Bera, "Solution of an extraordinary differential equation by Adomian decomposition method," Journal of Applied Mathematics, vol. 2004, no. 4, pp. 331-338, 2004.

[28] S. Saha-Ray, B. P. Poddar, and R. K. Bera, "Analytical solution of a dynamic system containing fractional derivative of order one-half by adomian decomposition method," Journal of Applied Mechanics, vol. 72, no. 2, pp. 290-295, 2005. 
[29] S. Saha-Ray and R. K. Bera, "An approximate solution of a non-linear fractional differential equation by Adomian decomposition method," Applied Mathematics and Computation, vol. 167, no. 1, pp. 561-571, 2005.

[30] S. Saha-Ray and R. K. Bera, "Analytical solution of the Bagley Torvik equation by Adomian decomposition method," Applied Mathematics and Computation, vol. 168, no. 1, pp. 398-410, 2005. 

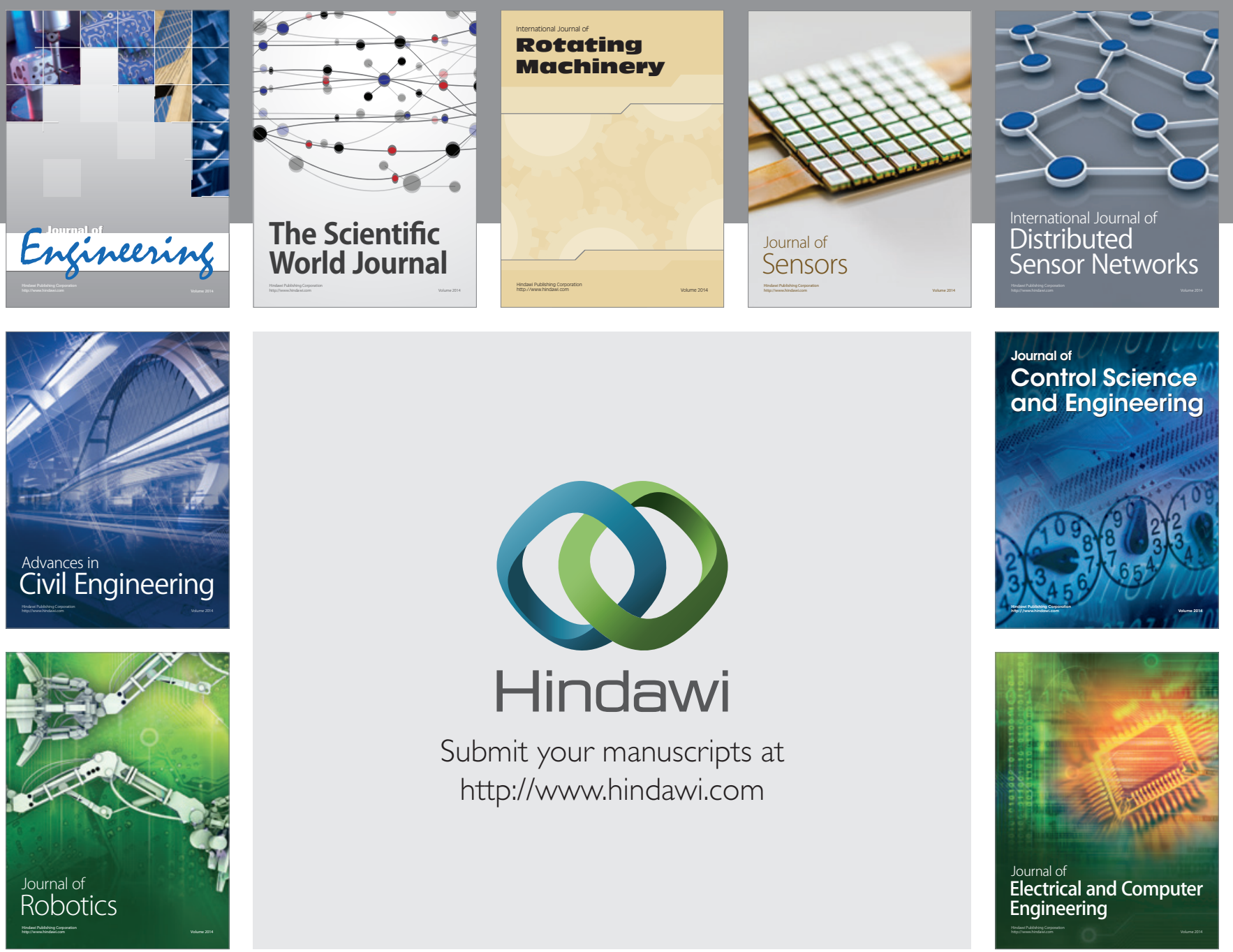

Submit your manuscripts at

http://www.hindawi.com
